\title{
MASS SPECTROSCOPY IN POLYMER RESEARCH
}

\author{
Jale Hacaloglu and Talat Yalcin
}

\subsection{INTRODUCTION}

Mass spectrometers are currently available in numerous configurations with various ionization techniques and mass analyzers. For a specific polymer sample, an optimal choice should be done depending on the information sought. In practice, no single mass spectrometric technique can provide all necessary information for structural, compositional, and thermal and oxidative degradation characteristics of polymeric samples. The analyzer used determines the limits of the sensitivity and resolution and the mass range, whereas the type of ionization source specifies the information that can be gained.

Mass spectroscopy in polymer research either involves degradation of the sample prior to analysis or vaporization/ionization of high molecular weight molecules into the mass spectrometer. The application of mass spectroscopy in polymer research was almost limited to degradation prior to mass spectroscopic analysis until the introduction of soft ionization techniques, matrix-assisted laser desorption/ionization, (MALDI), and electrospray ionization, (ESI). With the development of soft ionization methods, mass spectrometry (MS) has become a significantly powerful technique for characterization of polymers. Today, direct laser or matrix-assisted laser desorption/ionization (LDI and MALDI) and ESI and its variations-desorption electrospray and electrosonic spray ionizations (DESI and ESSI) - introducing little or no fragmentation are among the most commonly used ionization techniques. Polymer research applications involve structural characterizations such as identification of polymer chain repeat units, end groups, linear or cyclic geometry, com- position, sequence, presence and nature of additives, and determination of absolute molecular weights and molecular weight distributions of synthetic polymers of various chemical types [1,2].

The MALDI method involves cocrystallization of the analyte with a low molecular weight matrix that can absorb the energy of a laser light pulse. Absorption of the laser energy causes the matrix to undergo a phase transition and by intermolecular interactions the polymer sample is ionized within the plume, usually by proton transfer. Desorption/ionization techniques are performed without a matrix to overcome the limitations of MALDI for samples with relatively low molecular mass (<3000 Da). The matrix-assisted laser desorption/ ionization time-of-flight mass spectrometry (MALDITOFMS) combined with the ultrasonic degradation technique (UD/MALDI-TOFMS) can also be used for the analysis of synthetic polymer structures with high molecular weights $[3,4]$.

ESI is another method suitable for high molecular weight compounds, in which the sample is dissolved in a preferably polar solvent and can efficiently be interfaced with separation techniques such as liquid chromatography, LC [5]. However, the multiply charged ions generated can clutter the spectra. Both DESI and ESSI use high flow rates of nebulizing gas to produce small initial droplets which facilitate solvent evaporation. DESI is conducted under ambient conditions by exposing the solid-phase sample to a pneumatically assisted electrospray and the charged solvent droplets impact the solid surface and transport desorbed sample ions toward the MS inlet. In general, ESSI is used to analyze solution-phase polymers.

Mass Spectrometry Handbook, First Edition. Edited by Mike S. Lee.

(c) 2012 John Wiley \& Sons, Inc. Published 2012 by John Wiley \& Sons, Inc. 
Pyrolytic fingerprints of the polymeric parent materials are obtained by pyrolysis, the thermal degradation of a compound in an inert atmosphere or vacuum. Pyrolysis leads either low or high mass pyrolyzates depending on the thermal stability of the polymer, pyrolysis temperature, or heating rate. The two main pyrolysis mass spectrometry (py-MS) techniques, pyrolysis gas chromatography-mass spectrometry (py-(GCMS)) and direct pyrolysis mass spectrometry, (DP-MS), give information not only about the thermal stability and degradation pathways but also about composition, microstructure, and additives and are applied widely in polymer research. Py-MS techniques are still preferential for too large or too polar or unionizable synthetic polymers that cannot be analyzed directly by MS. Thermogravimetry mass spectrometry, (TG-MS) is another commercially available technique used for the purpose of thermal characterization of polymers. Stability of polymers regarding not only thermal but also photooxidative and other treatments is of great importance in the investigation of lifetime and durability of polymers in various applications.

The use of secondary ion mass spectrometry (SIMS) enables surface and interfacial characterization of polymers, copolymers, blends, and composites. Time-of-flight secondary ion mass spectrometry (TOF-SIMS) is applied for the investigation of the surface composition of adsorbed layers and molecular conformation of adsorbed block copolymers. Diffusion phenomena, adhesion, and chemical reactions at the interfaces of polymeric materials that need in situ analysis as a function of depth can also be analyzed by SIMS.

Whatever the purpose of the analysis, the mass spectrometer has to be prepared first for the analysis:

1. Turn the MS instrument on and wait until it is ready.

2. Tune/autotune the MS according to the manufacturer's specifications.

Note: The detector voltage influences the sensitivity of the detector, but if the detector voltage is too high, a loss in the signal-to-noise ratio may result because of the increase in background noise.

3. Calibrate the mass spectrometer considering the mass range.

4. Obtain a background spectrum or make a blank injection if chromatography is used.

Note: Record the spectrum without any sample using the experimental conditions selected for the analysis. No peaks other than background gases should be present in the background spectrum. If some extra peaks are present, then check the system to determine if any contamination is present. If the mass spectrometer is contaminated, then clean the system according to the guidelines given by the manufacturer.

5. Analyze samples and process the data.

\subsection{STRUCTURAL ANALYSIS}

MS has progressed to become a powerful technique for identification of polymer chain repeat units, end groups, linear or cyclic geometry, composition, sequence, presence, and nature of additives. The important physical properties of a polymer can be determined by its end group composition. Moreover, the end groups provide further information such as the initiation and termination chemistry of the polymerization reaction. In general, time-of flight mass spectrometers are used for the purpose with significantly large mass ranges. Among the soft ionization methods, MALDI and ESI techniques are the major ionization sources. The high resolution and high accuracy provided by Fourier transform mass spectrometry (FTMS) can be used to confirm the presence of a repeating unit and to determine the end groups of polymers.

\subsubsection{Laser Desorption/Ionization Mass Spectrometry}

Laser desorption generates high mass ions by irradiating a solid with a pulsed laser beam. In the case of matrix-assisted laser desorption, a solid mixture of an analyte is dissolved in a suitable matrix compound and the laser pulse desorbs and indirectly ionizes the analyte molecules. The selection of appropriate MALDI matrix, cationization salt, sample concentration, and sample preparation technique are critical success factors for obtaining a reliable mass spectrum that influence the polymer distribution. An alternative technique used to eliminate matrix and cationization salt effects is direct LDI, which is generally used for polymer samples with relatively low molecular weights.

46.2.1.1 Matrix-Assisted Laser Desorption/Ionization Mass Spectrometry (MALDI-MS) In practice, MALDI analysis consists of two steps: sample preparation and mass spectral analysis.

A general procedure for polymer characterization is as follows:

1. Prepare a solution of the polymer sample in a common solvent such as tetrahydrofuran (THF), chloroform, acetone, methanol, or water. 
2. Prepare a solution of sodium or potassium salt in ethanol, methanol, or water. Typical salt:matrix concentration ratio is $1: 10^{3}$.

3. Prepare about $0.10-0.20 \mathrm{M}$ matrix solution in the solvent used for sample preparation. Typical analyte:matrix concentration ratio is $1: 10^{4}$.

4. Prepare a mixture of analyte, salt, and matrix solution in a ratio of about 1:1000:10,000. Transfer $1.0 \mu \mathrm{L}$ of the mixture to the MALDI probe and allow air drying.

5. Prepare the MS instrument according to the manufacturer's specifications and general procedures.

6. Transfer the sample target into the vacuum for analysis.

7. Use the laser to generate ions.

8. Adjust the laser power slightly above the threshold of the desorption/ionization process.

9. Collect 100-200 shots for signal averaging.

The following protocol describes a mass spectrometric method for determining the end groups of a polystyrene (PS) sample [6].

\section{Material}

Polystyrene (average molecular weight 29,000 Da)

\section{Procedure}

1. Prepare $1.0 \mathrm{mg} / \mathrm{mL}$ solution of sample in chloroform or THF.

2. Prepare $0.15 \mathrm{M} \mathrm{AgTFA}$ (or $\mathrm{AgNO}_{3}$ ) in ethanol.

3. Prepare $0.15 \mathrm{M}$ dithranol, the matrix, in chloroform.

4. Mix $5 \mu \mathrm{L}$ of the polymer solution, $0.5 \mu \mathrm{L}$ of AgTFA, $44.5 \mu \mathrm{L}$ of the matrix solution, and $50 \mu \mathrm{L}$ of chloroform (or THF).

5. Prepare the sample target and collect the spectra as described above using $337 \mathrm{~nm}$ photon beam from the pulsed nitrogen laser.

Figure 46.1 contains a MALDI mass spectrum of a PS 29,000 Da. The spectrum features wellresolved styrene oligomer peaks. The mass difference between the two adjacent peaks from the main distribution is $104 \mathrm{Da}$, which corresponds to the mass of the repeat unit of styrene $\left[-\mathrm{CH}_{2}-\mathrm{CH}(-\right.$ $\mathrm{Ph})]$. The result confirms that the analyte is styrene-based polymer.

6. Conduct the repeat-unit and end-group analyses using:

$$
\begin{aligned}
& M_{\text {end }}=M_{\text {peak }}-M_{e}-M_{C a}-\mathrm{nM}_{r e}, \\
& \text { for cationized species }[M+\text { Cation }]^{+},
\end{aligned}
$$

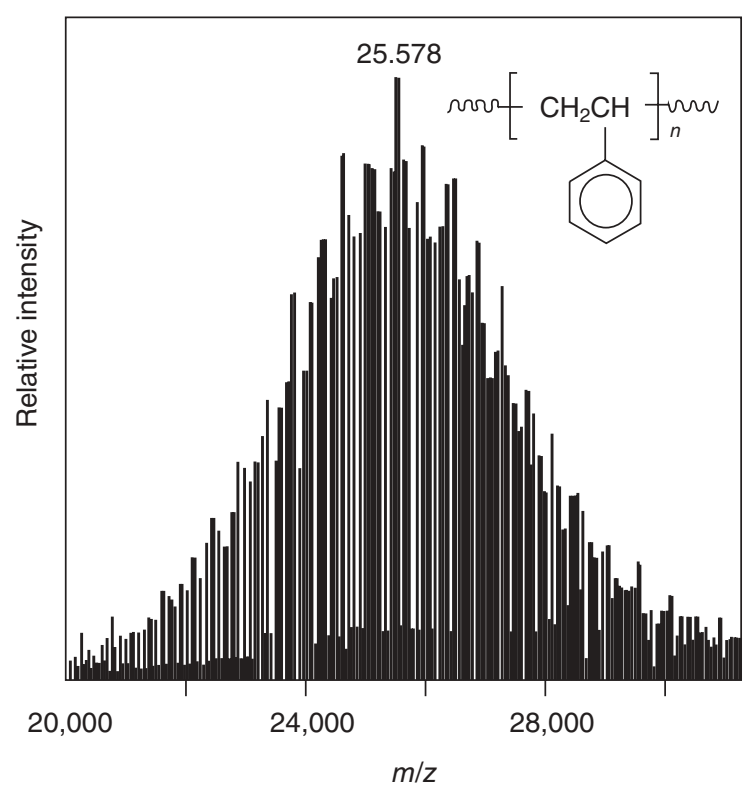

FIGURE 46.1 Structure and positive ion MALDI mass spectrum of a PS.

where $\mathrm{M}_{\mathrm{end}}$ is the sum of the end group masses, $M_{\text {peak }}$ is the mass of the selected peak, $M_{e}$ is the mass of an electron (actually negligible) $\mathrm{nM}_{\mathrm{re}}$ is $\mathrm{n}$ times the mass of the repeat unit, and $\mathrm{M}_{\mathrm{Ca}}$ is the cation mass.

For the present example:

$$
\begin{aligned}
\mathrm{M}_{\text {peak }} & =25,578 \mathrm{Da}, \\
\mathrm{M}_{\mathrm{Ca}} & =107.96 \mathrm{Da}, \\
\mathrm{M}_{\text {end }} & =25,578-107.96-104.15 \times \mathrm{n} .
\end{aligned}
$$

Trial-and-error method gives $\mathrm{M}_{\text {end }}=57.4 \mathrm{Da}$ $\left(\mathbf{C}_{4} \mathbf{H}_{9}\right.$ or $\left.\mathbf{C H}_{3}-\mathbf{C H}\left(\mathbf{C H}_{3}\right)-\mathbf{C H}_{2}\right)$ for $\mathrm{n}=244$ $(\mathrm{n} \sim(25,578-107.96) / 104.15=244,5515$; thus, $\mathrm{n}$ can be approximated to 244 ).

Critical Parameters The MALDI technique can be applied for polymers with molecular mass larger than $1500 \mathrm{Da}$. Most of the synthetic polymers having heteroatoms such as polyester or polyamides and low polarity polymers, such as PS, polybutadiene , and polyisoprene are easily ionized by addition of a sodium or potassium salt. On the other hand, polyethylene and polypropylene are very difficult to ionize by the MALDI process due to the absence of functional groups, such as a double bond, in their molecular structure. Therefore, it is necessary to add a cationizing agent to the matrix-polymer mixture. The matrix and cationizing agent selection as well as the sample preparation are very important parameters to obtain a suitable polymer signal. 
The choice of a specific matrix is mainly experimental. The analyte must be miscible with the matrix and they must cocrystallize. For positive ionization acidic, proton donor matrixes are favored, and for negative ionization mode, basic matrixes. "Hot spots" may be generated during cocrystallization if a nonuniform mixture is produced. Heterogeneity of the sample poses a major difficulty and in such a case the position of the laser beam may influence the spectra. In general, the selection of appropriate MALDI matrix, cationization salt, and sample concentration and sample preparation technique are critical success factors for obtaining a reliable mass spectrum that influence the polymer distribution.

Laser energy and delay time are parameters that influence how much sample is desorbed and cationized in the MALDI process. The Einzel lens and extraction voltages influence the ion envelope.

In general, spectroscopic or chromatographic data and/or knowledge are required to elucidate the correct end groups.

\subsubsection{Direct Laser Desorption/Ionization Mass} Spectrometry (LDI-MS) An alternative technique used to eliminate matrix and cationization salt effects is LDI.

A general procedure for polymer characterization by LDI-MS is as follows:

1. Prepare a solution of the sample by using common solvents such as chlorobenzene, benzene, toluene, or methanol.

2. Suspend the metal powder in isopropyl alcohol or dissolve the metal salt in proper solvent such as water.

3. Transfer about $1 \mu \mathrm{L}$ of metal salt or metal powder solution onto to the stainless sample target to form the first layer.

4. Deposit the sample solution directly onto to the metal salt or metal powder as a second layer and allow air drying.

5. Prepare the MS instrument according to the manufacturer's specifications and general procedures.

6. Transfer the sample target into the vacuum for analysis.

7. Use a laser to generate ions

8. Adjust the laser power slightly above the threshold of the desorption/ionization process.

9. Collect 100-200 shots for signal averaging.

The following protocol describes the application of LDI and MALDI in characterization of conjugated polymers [7].

\section{Materials}

poly(9,9-dioctylfluorene) (PF) $M_{w}=6800$ and $M_{n}$ 5000 , polysdispersity 1.36

poly(3-octylthiophene) (PT) $\mathrm{M}_{\mathrm{w}}=5100$ and $\mathrm{M}_{\mathrm{n}} 2600$, polysdispersity 1.96

\section{Procedure}

1. Turn on the mass spectrometer and calibrate using mass standards according to the manufacturer's specifications.

2. Analyze the sample using the operational parameters given in Table 46.1.

The MALDI and LDI spectra of PF and PT are shown in Figures 46.2 and 46.3, respectively.

3. Compare the spectra of MALDI-TOFMS with those of laser desorption/ionization time-of-flight mass spectrometry (LDI-TOFMS) to differentiate the peaks of the matrix and matrix-cationization salt.

Note: In the present example, the peaks of the matrix $(\mathrm{m} / \mathrm{z} 226)$ and the matrix-silver complex $(\mathrm{m} / z \quad 226+107=333)$ are found in Figures 46.2A and 46.3A, but absent in Figures 46.2B and $46.3 \mathrm{~B}$.

4. Find peak series and count out the masses of the repeat unit of each polymer by the information of different end groups.

TABLE 46.1 Operational Parameters Used for Analysis of Conjugated Polymers by LDI and MALDI-TOFMS

\begin{tabular}{ll}
\hline Sample & PF, PT $(5 \mathrm{mg} / \mathrm{mL})$ \\
Solvent & THF \\
Sampling rate digitizer & $2 \mathrm{GHz}$ \\
Pulse width & $3 \mathrm{~ns}$ \\
Power & 133 and $158 \mu \mathrm{J}$ for MALDI \\
& for PF and PT, respectively \\
& 142 and $168 \mu \mathrm{J}$ for LDI for PF \\
& and PT, respectively \\
LDI & $2 \mu \mathrm{L}$ \\
$\quad$ Sample & \\
MALDI & $1,8-$ dihydroxy- $9(10 \mathrm{H})-$ \\
$\quad$ Matrix & anthracenone $(20 \mathrm{mg} / \mathrm{mL})$ \\
& Silver trifluoroacetate $(5 \mathrm{mg} /$ \\
$\quad$ Cationization salt & $\mathrm{mL})$ \\
$\quad$ & $2: 20: 2 \mu \mathrm{L}$ \\
$\quad$ Sample:matrix:salt & TOF \\
Mass spectrometer & $20 \mathrm{kV}$ \\
Accelerating voltage & $-2.5 \mathrm{kV}$ \\
Pulsed voltage & $25 \mathrm{kV}$ \\
Reflectron voltage &
\end{tabular}




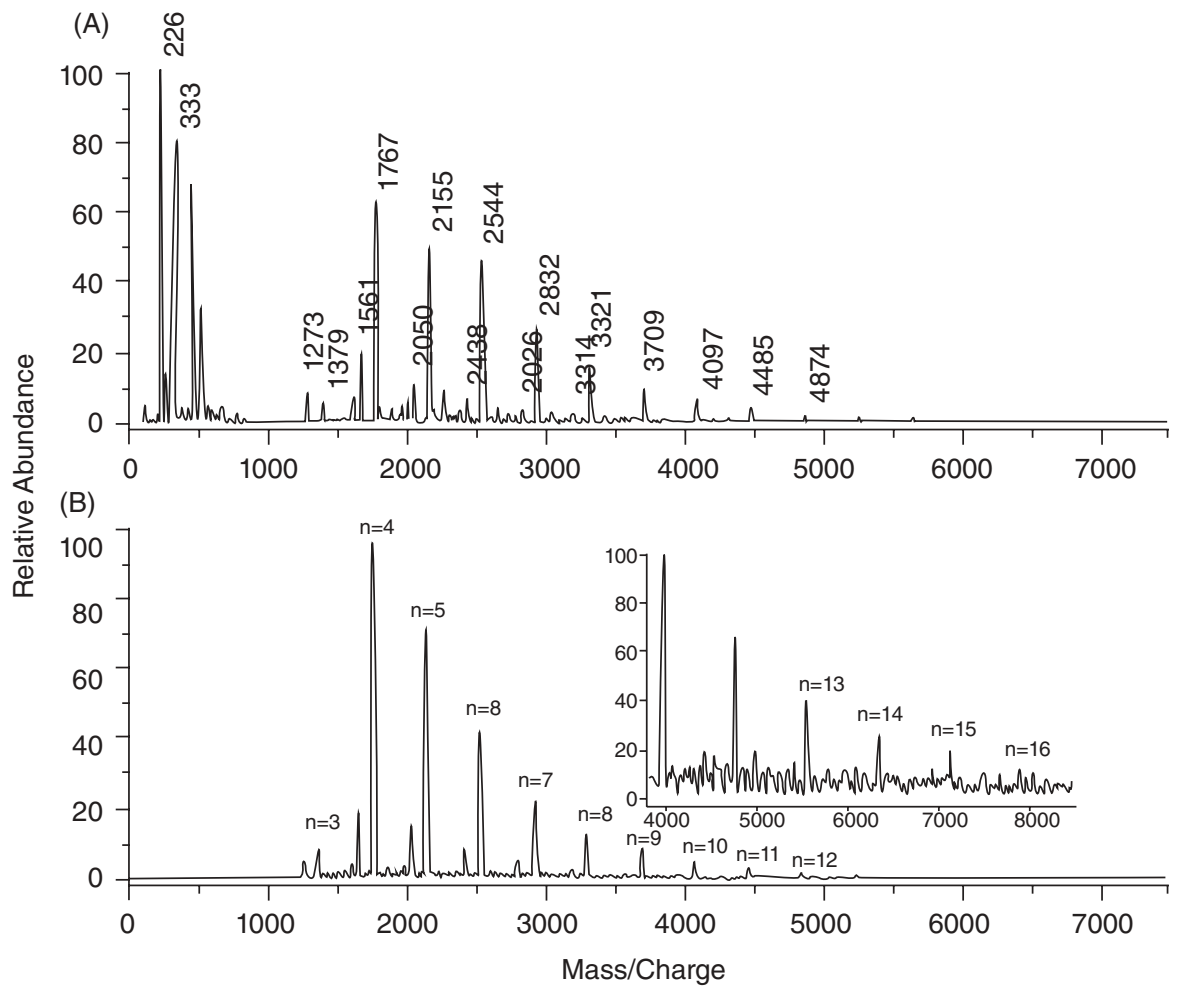

FIGURE 46.2 Mass spectra (in reflectron mode) of PF. (A) MALDI mass spectrum of PF; (B) direct LDI mass spectrum of PF (the inset shows the enlarged high mass region from $\mathrm{m} / \mathrm{z} 4400$ to 6700).

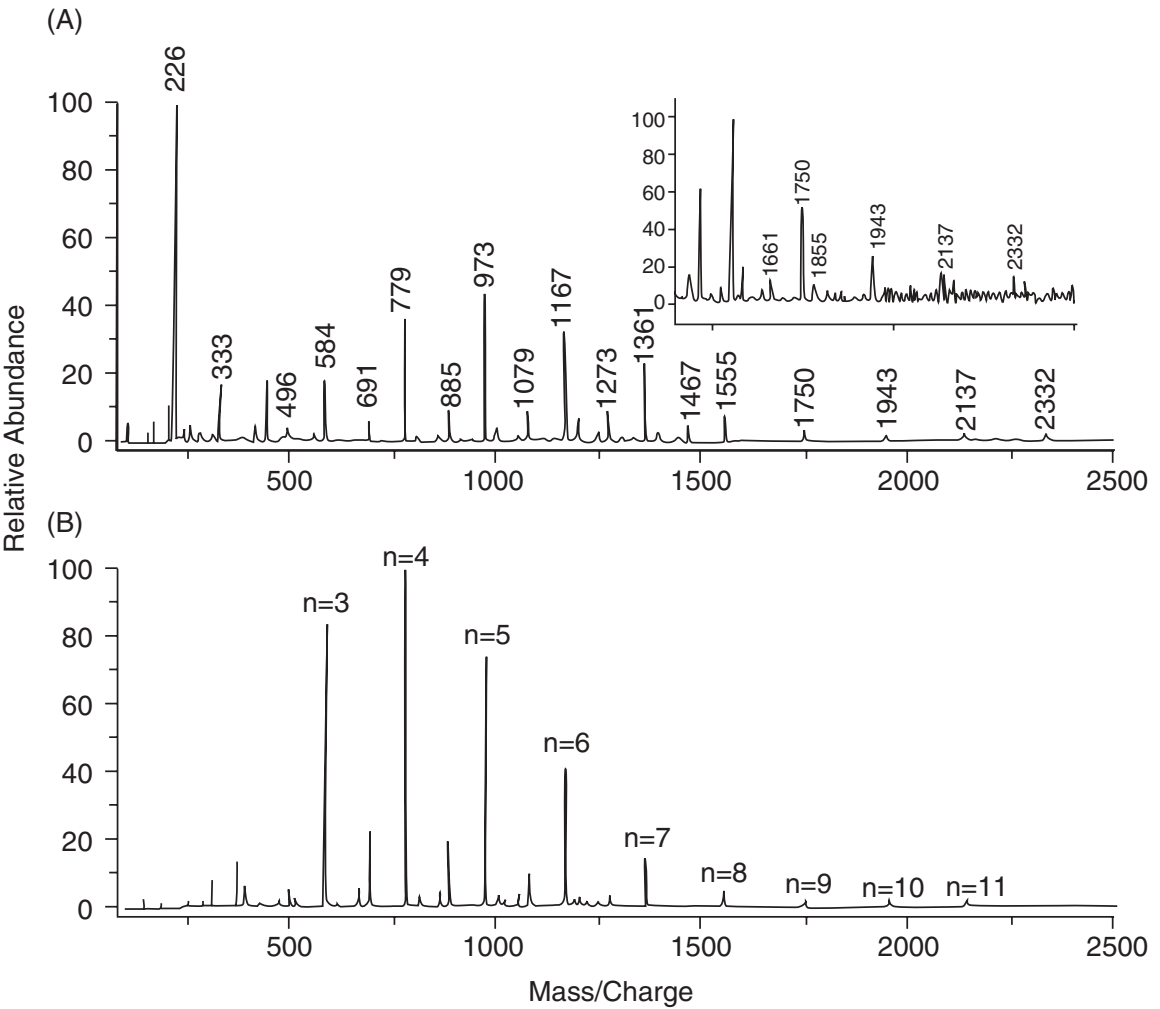

FIGURE 46.3 Mass spectra (in reflectron mode) of PT. (A) MALDI mass spectrum of PT; (B) direct LDI mass spectra of PT (the inset shows the enlarged high mass region from $\mathrm{m} / \mathrm{z} 1400$ to 2500). 
5. Conduct the repeat-unit and end-group analyses using:

$$
\mathrm{M}_{\text {end }}=\mathrm{M}_{\text {peak }}-\mathrm{M}_{\mathrm{e}}-\mathrm{nM}_{\mathrm{re}} \text {, }
$$

for radical ions $[\mathrm{M}]^{+}$by photoionization

and

$$
\begin{aligned}
& M_{\text {end }}=M_{\text {peak }}-M_{e}-M_{C a}-\mathrm{nM}_{\mathrm{re}}, \\
& \text { for cationized species }[\mathrm{M}+\text { Cation }]^{+},
\end{aligned}
$$

where $\mathrm{M}_{\text {end }}$ is the sum of the end group masses, $M_{\text {peak }}$ is the mass of the selected peak, $M_{e}$ is the mass of an electron (actually negligible), $\mathrm{nM}_{\mathrm{re}}$ is $\mathrm{n}$ times the mass of the repeat unit, and $\mathrm{M}_{\mathrm{Ca}}$ is the cation mass.

Note: Analysis of series of peaks observed in the spectra of PF-indicated benzyl alcohol and $\mathrm{H}$ end groups for both polymers indicated the following structures:

E- $(F) n-E n=16$, both end groups (E) are benzoyl alcohols

$\mathrm{n}=14$, one end group $(\mathrm{E})$ is $\mathrm{H}$ and the other is benzoyl alcohol

$\mathrm{E}-(\mathrm{T}) \mathrm{n}-\mathrm{E} \mathrm{n}=12$, both end groups are hydrogens $\mathrm{n}=9$, one end-group $(\mathrm{E})$ is $\mathrm{H}$ and the other is benzoyl alcohol

The results indicated that all polyfluorene chains are successfully end-capped at least at one end while only a small fraction of polythiophene chains is end-capped.

The LDI and MALDI-TOF mass spectra of the two polymers are nearly the same as those obtained from MALDI-in terms of mass values, resolution, and maximum detectable mass values. Although there is the same quality of mass spectra in LDI-TOFMS as in MALDI-TOFMS in this conjugated system, for LDI, as no interference due to matrix peaks exists, the sample peaks can more easily analyzed even in the low mass range.

Critical Parameters For LDI analysis, the samples must have an absorption in the region of laser radiation. The molecular weight of the samples ought not to be more than 10,000 Da. The polymers should have high thermal stability so that they get desorbed by absorbing laser energy but not by thermal decomposition.
46.2.1.3 Matrix-AssistedLaser Desorption/Ionization Fourier Transform Ion Cyclotron Resonance Mass Spectrometry (MALDI-FT-ICR-MS) The molecular weight of the end groups as well as the molecular weights of a number of oligomers of a polymer can be determined very precisely and accurately by Fourier transform mass spectrometers with high resolution that can help the assignment of the elemental composition. In particular, for copolymers that may have variable compositions and chain lengths, a high-resolution technique, such as Fourier transform ion cyclotron resonance mass spectrometry (FT-ICR-MS), is required to determine the structure and the oligomer distribution of the constituent subunits.

The following protocol describes a mass spectrometric method for the structural characterization of poly(neopentyl glycol-alt-isopthalic acid) homopolyester [8].

\section{Material}

Poly(neopentyl glycol-alt-isopthalic acid) homopolyester

\section{Procedure}

1. Prepare a solution of the polyester in acetone at a concentration of $1.0 \mathrm{mM}$.

2. Prepare a saturated solution (ca. $5 \mathrm{mM}$ ) of sodium trichloroacetate (NaTCA) in acetone.

3. Prepare $0.2 \mathrm{M}$ trans-3-indole acrylic acid (IAA) in acetone.

4. Mix $25 \mu \mathrm{L}$ of the polymer solution with $40 \mu \mathrm{L}$ of the matrix and $10 \mu \mathrm{L}$ of the salt solution.

5. Prepare the instrument according to the manufacturer's specifications and general procedures.

6. Apply the solution using electrospray deposition techniques.

7. Use 22-gauge metal hypodermic needle, suspend with a $1 \mathrm{~cm}$ gap above the titanium target and hold at a potential of $4.5 \mathrm{kV}$.

8. Analyze the sample.

Figure 46.4 shows the FT-ICR mass spectrum of a sample of polyester of neopentyl glycol and adipic acid (NPG-IPA). The mass spectrum of this simple alternating copolymer has some complexity due to the overlapping of the linear and cyclic oligomers and also due to the alcohol termination. Accurate monoisotopic mass assignment of each oligomer allows differentiation of cyclic oligomers from the linear oligomers.

With the application of the linear regression analysis method to polyester of NPG-IPA, residual mass can be 

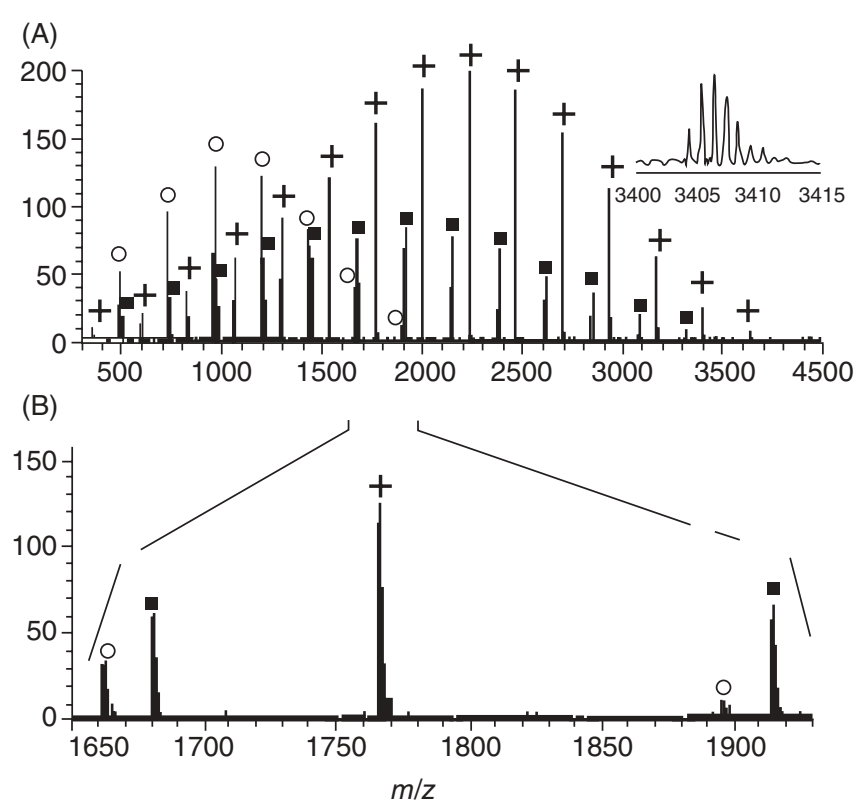

FIGURE 46.4 Poly(neopentyl glycol-alt-isopthalic acid) homopolyester mass spectrum synthesized with a 2:1 alcohol:acid ratio. This results in linear oligomers with alcohol end caps $(+)$ as well as normal linear oligomers with $\mathrm{H}$ - and $-\mathrm{OH}$ end groups ( $\mathbf{a}$ ) and cyclic species (o). (A) The complete spectrum with inset demonstrating typical resolution at $m / z=3450$. (B) An expansion of the spectrum showing cyclic and normal linear species at $\mathrm{n}=7$ and 8 and the alcohol endcapped oligomer at $\mathrm{n}=7$.

extracted as the masses of $22.9889\left(\mathrm{Na}^{+}\right), 41.00046$ $\left(\mathrm{H}_{2} \mathrm{O}+\mathrm{Na}^{+}\right)$, and $127.0735 \mathrm{Da}\left(\mathrm{C}_{5} \mathrm{H}_{12} \mathrm{O}_{2}+\mathrm{Na}^{+}\right)$for the cyclic, linear, and alcohol-saturated forms (calculated values are 22.9898, 41.0004, and 127.0735 $\mathrm{Da}$ for $\mathrm{Na}^{+}$, $\left[\mathrm{H}_{2} \mathrm{O}+\mathrm{Na}^{+}\right]$, and $\left[\mathrm{C}_{5} \mathrm{H}_{12} \mathrm{O}_{2}+\mathrm{Na}^{+}\right]$, respectively). The repeating unit mass can be found as $234.0892 \mathrm{Da}$ (calculated value is $234.0892 \mathrm{Da}$ ).

The application of linear regression analysis method is shown in Figure 46.5 with the neopentyl glycol endcapped oligomers as an example. Extrapolation of the line obtained by plotting $\mathrm{m} / \mathrm{z}$ versus degree of polymerization, $\mathrm{n}$, to $\mathrm{n}=0$ gives the exact mass of the repeat unit. Forcing lines with slope equal to the calculated mass of the correctly assigned repeat units mass through the data points results in several intercepts (inset) which are averaged to yield an order of magnitude better mass accuracy in the end group assignment.

\subsubsection{Electrospray Ionization Mass Spectrometry, ESI-MS}

ESI, in the most general sense is a method in which a solution is sprayed from a small tube into a strong electric field generating multiply charged ions. Besides the

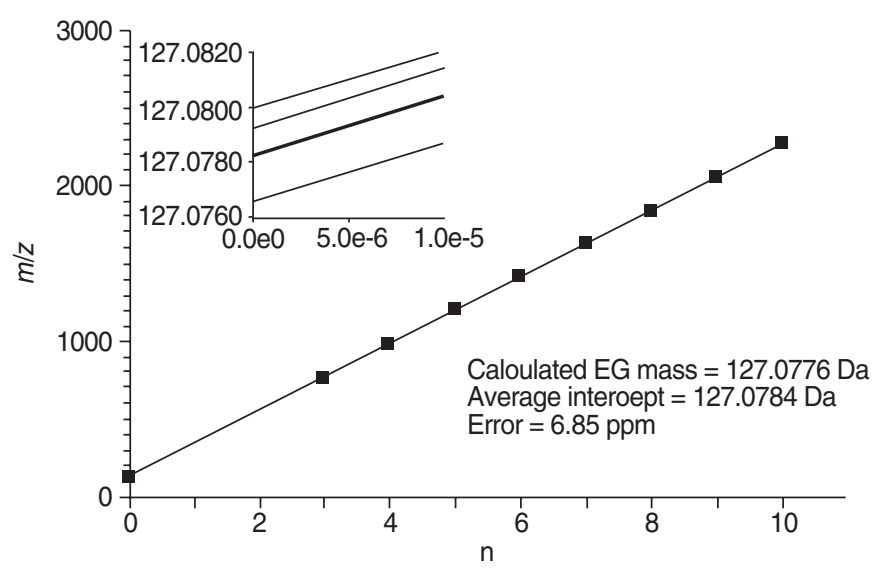

FIGURE 46.5 Linear regression of measured mass to charge versus degree of polymerization yields repeat unit mass of sufficient accuracy to assign an elemental composition for the repeat mass but not for the end group mass.

common ESI source used in polymer analysis, DESI and ESSI, as variants of electrospray source, are constructed to facilitate solvent evaporation are used.

46.2.2.1 Electrospray Ionization Tandem Mass Spectrometry (ES-MS/MS/MS) High-energy collisioninduced dissociation (CID) spectra of source-induced fragment ions can be used for end group structure characterization. Chain cleavage appears to be the dominant process in CID and further dissociation of the fragment ions after mass selection should potentially generate structural information on the end group. By comparing the CID spectra of fragment ions with those of known chemicals, it is possible to unambiguously determine the end group structures.

A general procedure for end group analysis is as follows

1. Prepare a solution of the sample by using common solvents such as acetonitrile methanol and water. Metal salt or proton donor species should also be added to the solution for cationization or protonation of the polymer.

2. Deliver the sample solution $(50-100 \mu \mathrm{M})$ from a syringe to the ESI source at a flow rate of $5-10 \mu \mathrm{L} /$ min using the continuous infusion method or from through the column to separate the mixture of oligomers to the ESI source with the same flow rate.

3. Adjust the voltage difference between the atmospheric plate and the skimmer in the ESI interface to control the source fragmentation of the polymer ions. 
4. Accelerate the precursor ions by a voltage of $4000-4700 \mathrm{~V}\left(\mathrm{~V}_{\mathrm{Ac}}\right)$.

5. Select the precursor ions by a trisector (electrostatic/magnetic/electrostatic) EBE mass spectrometer and introduce them into the collision cell floated at around $3600 \mathrm{~V}\left(\mathrm{~V}_{\mathrm{CID}}\right)$. The kinetic energy of the ions with charge $\mathrm{z}$ is $\left(\mathrm{V}_{\mathrm{Ac}}-\mathrm{V}_{\mathrm{CID}}\right) \mathrm{zeV}$.

6. Among the oligomers with different charge states, the ones at a charge state of +4 or greater should be chosen, as oligomers with lower charge states do not fragment as efficiently as the high charged ions.

7. Determine the series of peaks separated by one or more monomer units.

8. Obtain the CID spectrum of the corresponding ions.

9. Identify the maximum $\mathrm{m} / \mathrm{z}$ ion that does not further dissociate by loss of monomer unit.

10. Compare the CID spectrum of this ion with those of known chemicals to determine the structure of the end group.

The following protocol describes a mass spectrometric method for determining end group structure of poly (oxyethylene) bis-(ephedrine) by ES-MS/MS/MS [9].

\section{Material}

poly(oxyethylene) bis-(ephedrine) (average molecular weight $3500 \mathrm{Da}$ )

\section{Procedure}

1. Prepare the solution for ESI MS analysis by dissolving the sample in a mixture of $80 \%(\mathrm{v} / \mathrm{v})$ acetonitrile and $20 \%(\mathrm{v} / \mathrm{v}) \quad 0.25 \mathrm{mM}$ ammonium acetate in water. Deliver the sample solution $(100 \mu \mathrm{M})$ from a syringe to the ESI source at a flow rate of $6 \mu \mathrm{L} / \mathrm{min}$ using the continuous infusion method.

2. Adjust the voltage difference between the atmospheric plate and the skimmer in the ESI interface to control the source fragmentation of the polymer ions.

3. Accelerate the precursor ions by a voltage of $4000 \mathrm{~V}$.

4. Select the precursor ions by EBE mass spectrometer and introduce them into the collision cell floated at $3600 \mathrm{~V}$. The kinetic energy of the ions with charge $\mathrm{z}$ is $400 \mathrm{zeV}$.

5. Among the oligomers with different charge states, the ones at a charge state of +4 are chosen.
Figure 46.6A illustrates the low mass region of the high-energy CID spectrum of an ion at $\mathrm{m} / z 1014$ from poly(oxyethylene) bis(ephedrine). A series of peaks from $\mathrm{G}_{\mathrm{n}}$ ions (i.e., $m / z$ at 148,192 , and 236) and $\mathrm{A}_{\mathrm{n}}$ ions (i.e., $m / z$ at $45,89,133$, and 177 ) were observed with a mass difference that corresponds to the monomer unit of the polymer (i.e., $44 \mathrm{Da}$ ). Figure $46.6 \mathrm{~B}, \mathrm{C}$ show the source fragmentation spectra of poly(oxyethylene) bis(ephedrine) obtained by using two different source voltages $\left(\mathrm{V}_{\mathrm{CID}}\right)$. A number of fragment ions are generated in source fragmentation; however, the ions from the $\mathrm{G}_{\mathrm{n}}$ series at $m / z, 148,192$, and 236 are preferentially formed (Figure 46.6B). As $\mathrm{V}_{\mathrm{CID}}$ increases, lower mass fragments become more intense.

The intense source fragment ion at $m / z 236$ shown in Figure $46.6 \mathrm{~B}, \mathrm{C}$ gives rise to a relatively simple CID spectrum (Figure 46.7A). The peak at $\mathrm{m} / \mathrm{z} 148$ is generated by the elimination of two monomer units from the $m / z 236$ ion. The same peak is also generated by the loss of one monomer unit from the $\mathrm{m} / \mathrm{z} 192$ ion (Figure 46.7B). The next source fragment ion in the series at $\mathrm{m} / \mathrm{z} 148$ generates a CID spectrum that closely resembles the low mass region of the CID spectrum from $\mathrm{m} / \mathrm{z} 192$ (Figure 46.7C). No intense fragment ion peak from the loss of $44 \mathrm{Da}$ is found in this spectrum. CID spectrum of the source-induced fragment ion at $\mathrm{m} / \mathrm{z} 148$ originated from ephedrine shown in Figure 46.7D is almost exactly similar to that of the ion with $\mathrm{m} / z, 148$ generated from the polymer. Thus, the $\mathrm{m} / z 148$ ion can tentatively be assigned as an ion from the end group of the polymer.

Critical Parameters ESI produces multiply charged species, thus theoretically unlimited mass range; however, this may result in difficulties in identifying the molecular ions. Source fragmentation is one of the major concerns in determining molecular weights of polymers by ESI. For polymers that do not fragment efficiently, variation of ESI conditions such as the change of solvents and types of cation affect the extent of source fragmentation. In ESI ionization, the relative abundance of various ions generated is not a real representation of the sample concentration. The sample should be soluble in a preferably polar solvent. The tendency toward multiple charging decreases in low polarity solvents. As a result, accessible mass range decreases, and discrimination among different molecular weight oligomers may not be generated. Thus, the choice of solvent is of significant importance especially for hydrophobic polymers, and thus, dissolution systems, usually mixtures of polar and nonpolar solvents, should be used. 
(A)

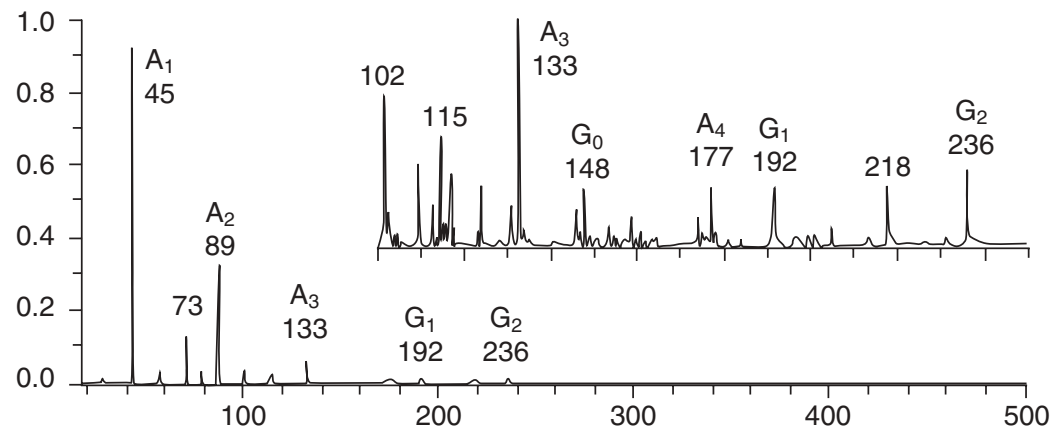

(B)

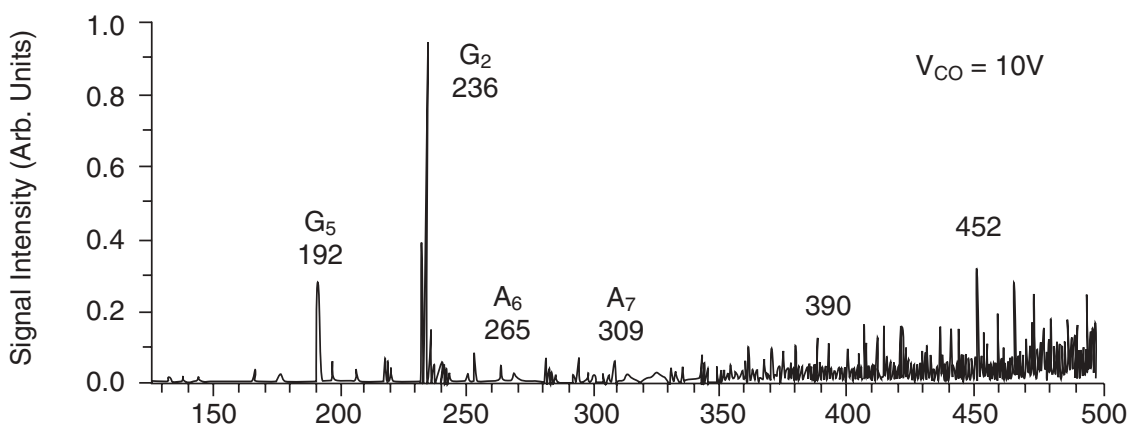

(C)

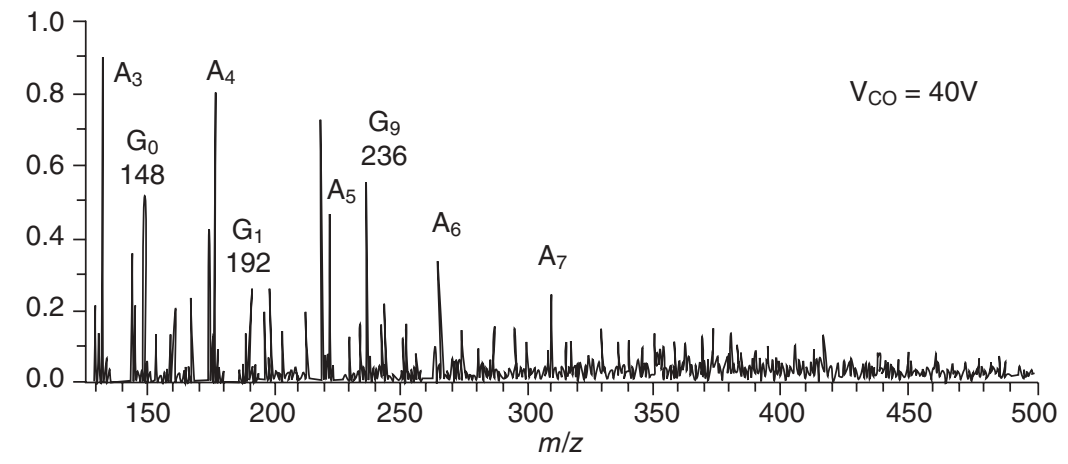

FIGURE 46.6 Fragment ion spectra of poly(oxyethylene) bis(ephedrine) obtained by using ESI tandem sector/time-of-flight mass spectrometry. (A) High-energy CID spectrum of an oligomer ion at $m / z, 1014$ with a +4 charge state; (B) source-induced CID spectrum of the polymer at $\mathrm{V}_{\mathrm{CID}}=10 \mathrm{~V}$; and $(\mathrm{C})$ source CID spectrum of the polymer at $\mathrm{V}_{\mathrm{CID}}=40 \mathrm{~V}$.

Mass discrimination, changes in the cone potential in the atmospheric interface, and accommodation of multiple charges by large molecules affect the reliability of measured molecular weights and molecular weight distributions especially for polydispersed polymers. Loss of resolution and raise in baseline may arise with the increase in number of possible types of ionic clusters for charges +4 and higher.

The sensitivity increases with increasing ionization efficiency and decreases with an increase in number of ion series generated.
Contamination of the tubes through which the sample is infused into the ESI needle may occur.

Online separation technique is not necessary for production of end group fingerprint spectra. This provides the advantage of simplicity and no need of compromising ESI condition and separation conditions. However, the presence of any low mass impurities in the polymer sample causes complications in spectral interpretation. Low mass impurities can be removed by offline separation by LC prior to tandem mass spectrometry (MS/MS) experiments. 

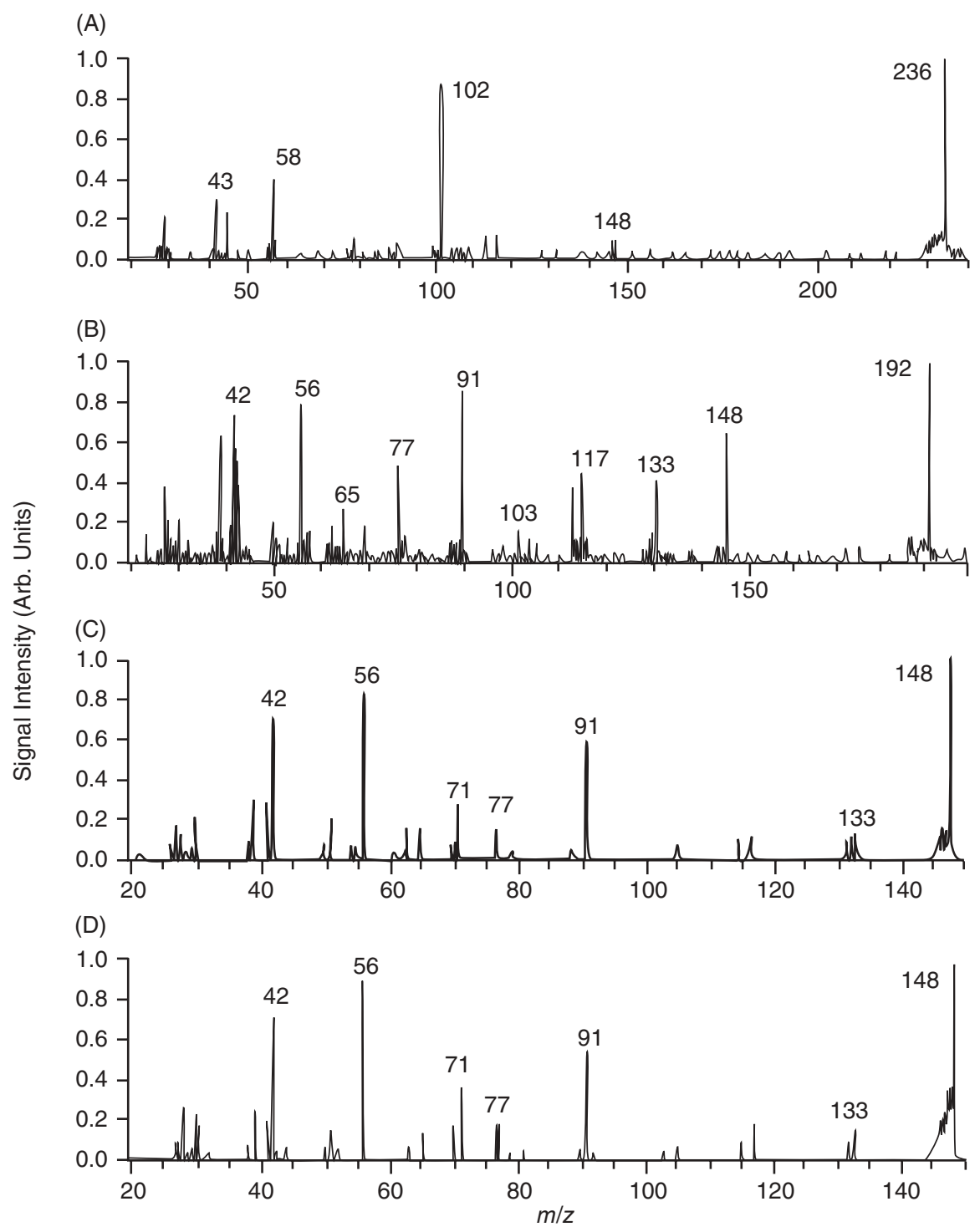

FIGURE 46.7 High-energy CID spectra of source-induced fragment ions at (A) $m / z$ 236, (B) 192, and (C) 148 derived from poly(oxyethylene) bis(ephedrine). (D) CID spectrum of the source-induced fragment ion at $\mathrm{m} / \mathrm{z} 148$ originated from ephedrine.

46.2.2.2 Liquid Chromatography Electrospray Ionization or Atmospheric Pressure Ionization Mass Spectrometry (LC/ESI-MS or LC/API-MS) LC attachment is an advantage due to online separation and characterization capability of the mixture of the polymer samples and blends. It is well known that chromatographic separation is tricky and is based on the properties of the samples. Thus, a general procedure that can be used for any type of polymer sample cannot be given. In general, ESI is used in liquid chromatographymass spectrometry (LC-MS) analyses. Atmospheric pressure chemical ionization (APCI) source can also be used especially for less polar samples.
A general procedure for LC-ESI analysis involves the following steps:

1. Prepare a solution of the sample using common solvents that can produce spray easily for ESI source, such as acetonitrile methanol and water. Metal salt or proton donor species should also be added to the solution for cationization or protonation of the polymer.

2. Deliver the sample solution $(50-100 \mu \mathrm{M})$ through the column to separate the mixture of oligomers to the ESI source at a flow rate of 5-10 $\mu \mathrm{L} / \mathrm{min}$. 
Note: In order to obtain a suitable chromatographic separation, different columns with $\mathrm{C}-18$ and C- 8 as the stationary phase can be used. Eluents (water or aqueous buffer as the aqueous phase, and acetonitrile or methanol as the organic phase) and gradient profiles should be explored.

3. For ESI, depending on the source, spray voltage can be set to 3000-4000 V, nebulizer gas can be set to $40.0-50.0 \mathrm{psi}$, drying gas flow rate can be set to $7.0-9.0 \mathrm{~L} / \mathrm{min}$, and drying gas temperature can be set to $350-365^{\circ} \mathrm{C}$.

4. For atmospheric pressure ionization (API) source, spray voltage can be set to $1200-2000 \mathrm{~V}$, nebulizer gas can be set to 40.0-50.0 psi, and drying gas flow rate can be set to $3.0-5.0 \mathrm{~L} / \mathrm{min}$. The nebulizer temperature should be set to $375^{\circ} \mathrm{C}$ and Corona current should be set as $375 \mathrm{nA}$ for APCI. The same drying gas temperature can be used.

5. Operate the mass spectrometer for both positive and negative modes.

6. Collect the full scan mass spectra and accumulate 20,000 and 5000 ions for positive and negative modes, respectively.

7. Duplicate each measurement to ensure reproducibility.

The following protocol describes a mass spectrometric method for characterization of a block copolymer consisting of methoxy poly(ethylene oxide) (mPEO), an - $\varepsilon$-caprolactone (CL) segment, and linoleic acid (LA), used as surfactant in water-based latex paints by liquid chromatography electrospray ionization (LC-ESI) or API-MS [10].

\section{Materials}

Block copolymer metoxy poly(etylene glycol) with an average molecular weight (MW) of 2000 (mPEG2000)

$\varepsilon$-Caprolactone $(\mathrm{CL})$

\section{Procedure}

1. Use C-8 column with a dimension of $2.1 \times 150 \mathrm{~mm}$ for reversed-phase chromatographic separation.

2. Use acetonitrile (solvent $\mathrm{B}$ ) in $20 \mathrm{mM}$ aqueous ammonium formate buffer ( $\mathrm{pH} \sim 3$, solvent $\mathrm{A}$ ) as eluent.

3. Apply the following gradient with a flow rate of $300 \mathrm{~mL} / \mathrm{min}: 2.0 \mathrm{~min}$ isocratic at $10 \% \mathrm{~B}(\mathrm{v} / \mathrm{v})$, increase solvent B to $95 \%$ over $38 \mathrm{~min}$, hold at $95 \% \mathrm{~B}$ for another $39 \mathrm{~min}$ and return to $10 \% \mathrm{~B}$ within 1 min (where a 10-min column equilibration is allowed).

4. Injection volumes of sample or blank solutions should be $5 \mathrm{~mL}$.

5. Analyze the sample by using ESI or API source according to the general procedure discussed above.

The possible backbone composition distribution of the polymer sample comprises unreacted mPEG, mPEO-pCLn copolymer, homopolymer of CL, and LA derivatives of all of these. All homologous series containing a CL block (mPEO-pCLn, mPEO-pCLn-LA, pCLn, pCLn-LA and cyclo-pCLn) are separated based on their number of CL units. In contrast, the separation of homologues based on the number of ethylene oxide (EO) units occurs only to a minor extent. Thus, relatively broad peaks containing the complete mPEO distribution for a given number of CL units can be obtained. The LC-MS chromatograms of the homologous polymer series contained in the block copolymer samples are shown in Figure 46.8. Examples of the corresponding mass spectra recorded with ESI or API sources are given. Products that contain the $\mathrm{mPEO}$ chain are easily differentiated (Figure $46.8 \mathrm{~A}^{*}, \mathrm{~B}^{*}$ ) from species without mPEO (Figure $46.8 \mathrm{C}^{*}-\mathrm{E}^{*}$ ). The individual homologous series do partially overlap (Figure 46.8A-E).

Figure 46.9 contains the mass spectra extracted from a specific chromatographic peak recorded using different ionization methods. The ESI(+) spectrum given in Figure $46.9 \mathrm{~A}$ shows the determination of mPEO-pCL with a specific value of $\mathrm{n}$ as doubly and triply charged ions. The polymer distribution observed in this spectrum results from the very poor chromatographic resolution of the mPEO distribution. In Figure 46.9B, in the $\mathrm{APCI}(+)$ spectrum, $\mathrm{mPEO}-\mathrm{pCL}_{\mathrm{n}}$ with the same value of $\mathrm{n}$ is observed, but as singly charged ions and partial overlap with a specific $\mathrm{pCL}_{\mathrm{n}}$ species are shown. The $\mathrm{pCL}_{\mathrm{n}}$ product is best observed in the mass spectrum obtained with APCI(-) (Figure 46.9C), where it dominates the spectrum.

Critical Parameters The combination of LC-MS provides online separation of polymer mixtures and blends. However, LC parameters can be changed for sample to sample and should be set for each run for all kind of polymers. Every different end group and the functional group of polymer can affect the separation quality of the polymer mixture. Different homologous series are best identified by different ionization techniques counterbalancing the incomplete resolution of the separation. The need for multiple analyses makes the overall analysis more time consuming. 


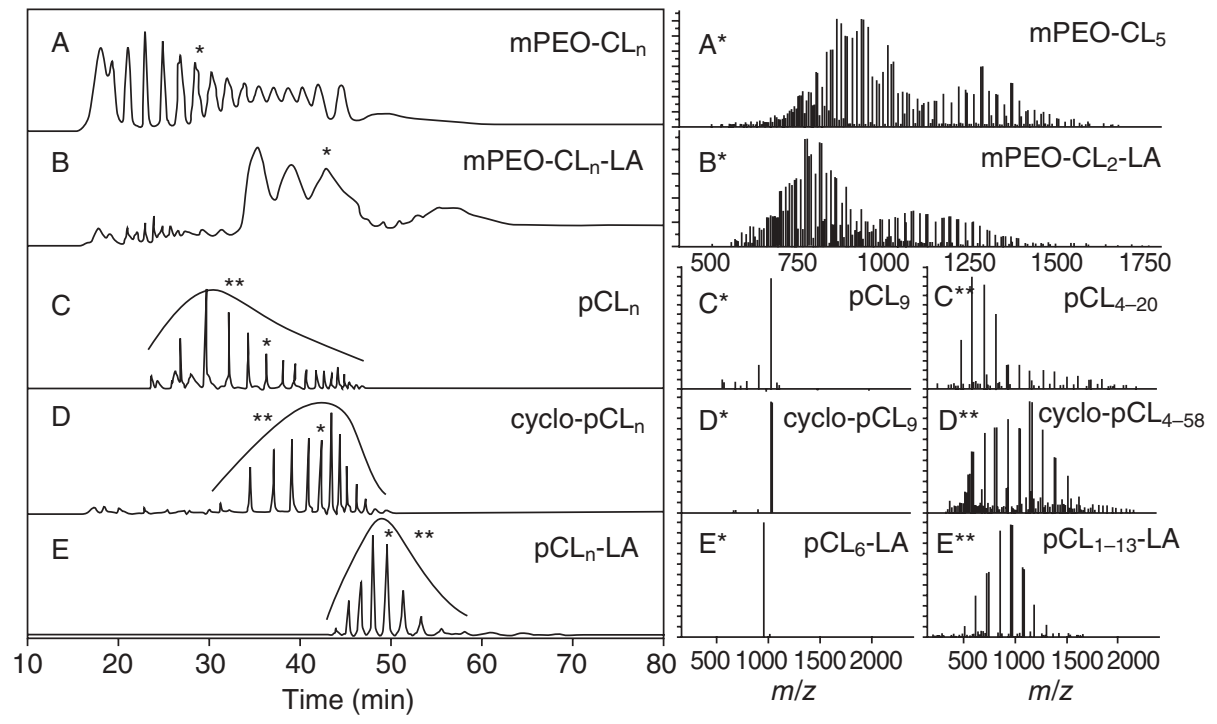

FIGURE 46.8 LC-MS chromatograms of the homologous polymer series contained in the block copolymer samples. (A) Total ion chromatogram of unfunctionalized polymer, recorded with ESI(+)-MS; (B) TIC of functionalized polymer, recorded with ESI(+)-MS; (C) multiple ion chromatogram (MIC) of 17 selected mass traces of unfunctionalized polymer, recorded with APCI(-)MS; (D) MIC of 14 selected mass traces of unfunctionalized polymer, recorded with APCI(+)-MS; and (E) MIC of 13 selected mass traces of functionalized polymer, recorded with APCI(-)-MS. Examples of the corresponding mass spectra are given for the marked peaks, as well as for the complete homologous series shown in (C), (D), and (E).

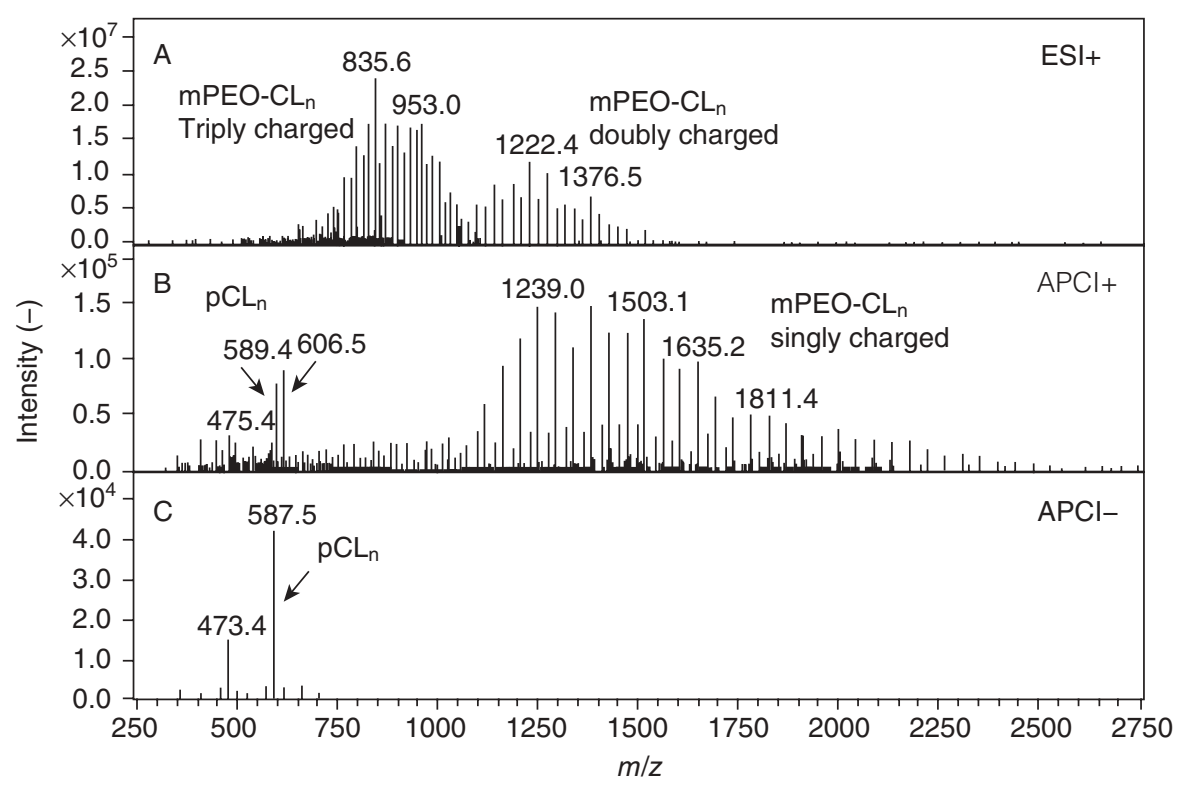

FIGURE 46.9 Mass spectra extracted from a specific chromatographic peak, recorded using different ionization methods: (A) $\operatorname{ESI}(+)$; (B) APCI(+); and (C) APCI(-).

\subsection{ABSOLUTE MOLECULAR WEIGHTS AND MOLECULAR WEIGHT DISTRIBUTIONS}

As MS yields exact molecular weights of individual molecules, no major inaccuracies are involved in the calculation of average molecular weights for oligomers and polymers with narrow molecular weight distributions.
However, accurate molecular weight distributions and reliable molecular weight averages of synthetic polymers that do not exhibit one uniform chain length can only be obtained in limited cases. Polymers may be complex mixtures with heterogeneity not only in size (molecular weight distribution), but also in chemical composition and end groups. Size-exclusion chromatog- 
raphy (SEC) combined with ESI-MS offers a solution for analysis of such complex mixtures.

In general, number-averaged molecular weight $\left(\mathrm{M}_{\mathrm{n}}\right)$, and weight-averaged molecular weight $\left(\mathrm{M}_{\mathrm{w}}\right)$ and polysdispersity index (PDI) can be calculated by application of MS techniques:

$$
\begin{aligned}
& \mathrm{M}_{\mathrm{n}}=\sum \mathrm{M}_{\mathrm{i}} \mathrm{N}_{\mathrm{i}} / \sum \mathrm{N}_{\mathrm{i}}, \\
& \mathrm{M}_{\mathrm{w}}=\sum\left(\mathrm{M}_{\mathrm{i}}\right)^{2} \mathrm{~N}_{\mathrm{i}} / \sum \mathrm{M}_{\mathrm{i}} \mathrm{N}_{\mathrm{i}},
\end{aligned}
$$

where

$\mathrm{M}_{\mathrm{i}}=$ mass of an observed ion $\mathrm{i}$,

$\mathrm{N}_{\mathrm{i}}=$ number of the $\mathrm{i}$ ions observed, and

$\mathrm{PDI}=\mathrm{M}_{\mathrm{w}} / \mathrm{M}_{\mathrm{n}}$ (polydispersity).

\subsubsection{LDI-MS}

46.3.1.1 MALDI-MS In general, the average molecular weights are calculated directly from ion intensity (or peak height) and ion masses in the mass spectrum using the equation given above. $\mathrm{N}_{\mathrm{i}}$ and $\mathrm{M}_{\mathrm{i}}$ represent signal intensity and mass at point i, respectively. For $M_{n}$ and $\mathrm{M}_{\mathrm{w}}$ calculation, peak area under each signal has been used instead of peak height. The protocol given for structural analysis of a polymer sample by MALDI (Section 46.2.1.1) can also be used for determining $M_{n}$, $\mathrm{M}_{\mathrm{w}}$, and PDI of the polymer. For the example given in Section 46.2.1.1, the $\mathrm{M}_{\mathrm{n}}$ and $\mathrm{M}_{\mathrm{w}}$ of PS (average molecular weight 29,000 Da) are calculated from the spectrum (Figure 46.1) as $\mathrm{M}_{\mathrm{n}}=26,250, \mathrm{M}_{\mathrm{w}}=26,500$, and PDI = 1.01 using the equations given above.

Critical Parameters The general critical parameters discussed for MALDI-MS analysis in Section 46.2.1.1 also apply for molecular weight analysis. A Gaussian, high-resolution oligomer distribution is important for the determination of the accurate $\mathrm{M}_{\mathrm{n}}, \mathrm{M}_{\mathrm{w}}$, and PDI by MALDI.

46.3.1.2 LDI-MS The following protocol describes a mass spectrometric method for determining $M_{n}, M_{w}$, and PDI of polyethylene by LDI-MS [11].

\section{Material}

Polyethylenes with varying molecular masses such as PE500, PE1000, and PE1100, 2100

\section{Procedure}

1. Prepare the solution for LDI TOF-MS analysis by dissolving the sample in chlorobenzene at $5 \mathrm{mg} /$ $\mathrm{mL}$ at ambient temperature.
Note: For samples with molecular masses above $2000 \mathrm{Da}$, heating is required to achieve the desired concentration $(5 \mathrm{mg} / \mathrm{mL})$.

2. Prepare the MS instrument for analysis according to the general procedures and manufacturer's specifications.

3. Follow the general procedures described in Section 46.2.1.2 for preparation of the sample target and for collecting data.

Figure 46.10 contains the LDI mass spectra of two PE samples that were obtained with a linear TOF mass spectrometer. For both cases, the mass difference between two adjacent peaks from the main distribution is $28 \mathrm{Da}$, which corresponds to the mass of the repeat unit of $-\mathrm{CH}_{2} \mathrm{CH}_{2}-$. From the masses and peak intensities of the main distribution after the removal of low mass ion contributions, $M_{n}$ and $M_{w}$ are found to be 1010 and 1103 Da, respectively, for PE1100 (Figure 46.10A) with a PDI of 1.095. For PE2100, $\mathbf{M}_{n}$ and $\mathrm{M}_{\mathrm{w}}$ are calculated to be 1815 and $2021 \mathrm{Da}$, respectively, with PDI of 1.113 (Figure 46.10B). For both samples, the LDI results are consistent with those of GPC (for PE1100: $\mathrm{M}_{\mathrm{n}}=$ $1050 \mathrm{Da}, \mathrm{M}_{\mathrm{w}}=1150 \mathrm{Da}$, and $\mathrm{PDI}=1.095$; and for PE2100: $\mathrm{M}_{\mathrm{n}}=1900 \mathrm{Da}, \mathrm{M}_{\mathrm{w}}=2150 \mathrm{Da}$, and PDI $=1.132$ ).

Note: Several low mass peaks with mass difference of 14 Da between adjacent peaks in the LDI spectrum of PE2100 were attributed to the fragment ions of PE oligomers or from the impurities present in the sample.
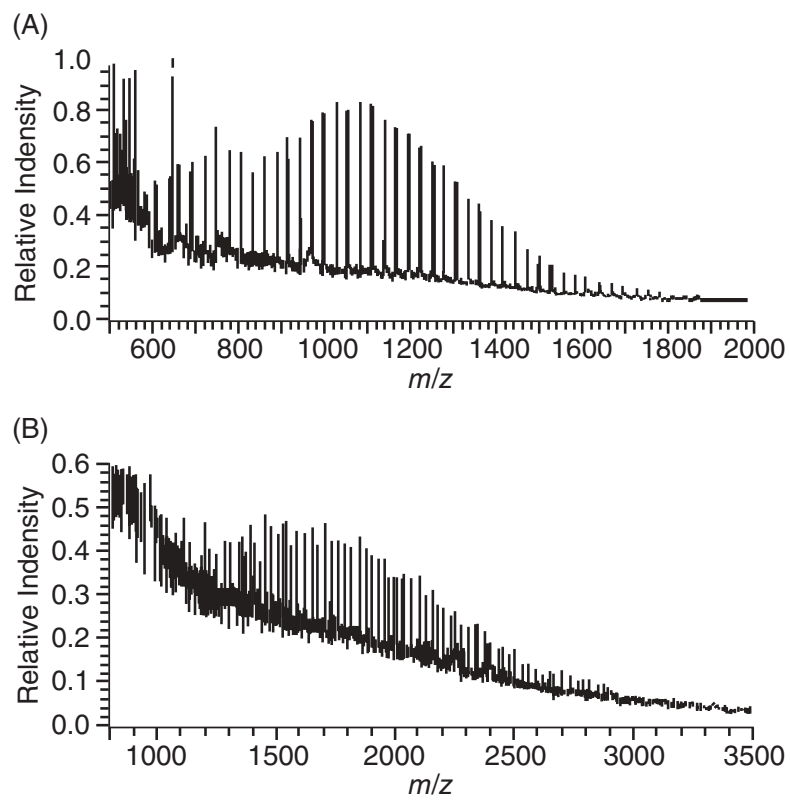

FIGURE 46.10 LDI mass spectra of (A) PE1100 and (B) PE2100, obtained by using a linear TOF mass spectrometer. 


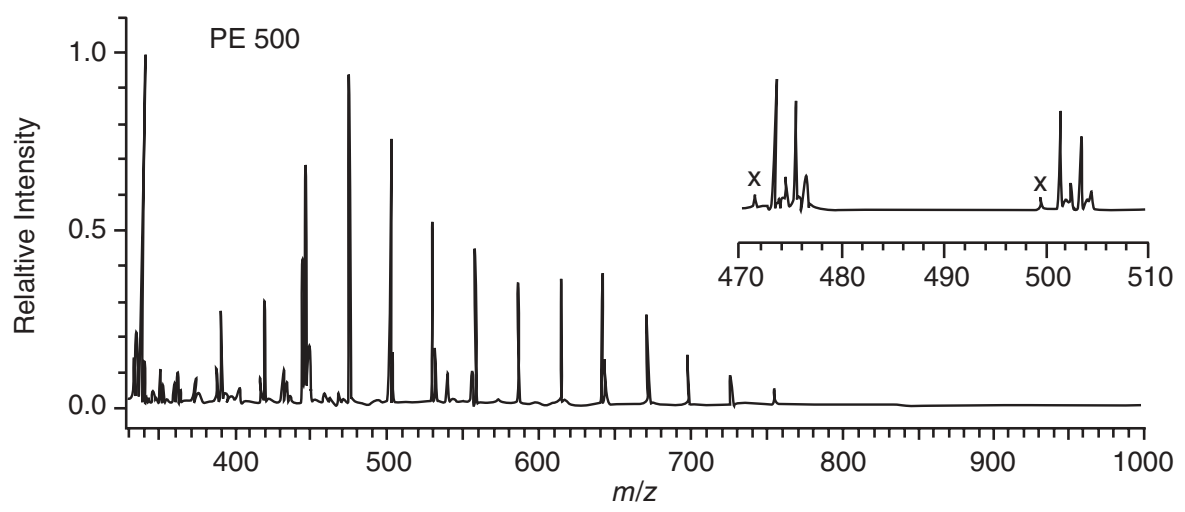

FIGURE 46.11 LDI mass spectra of PE500.

(A)

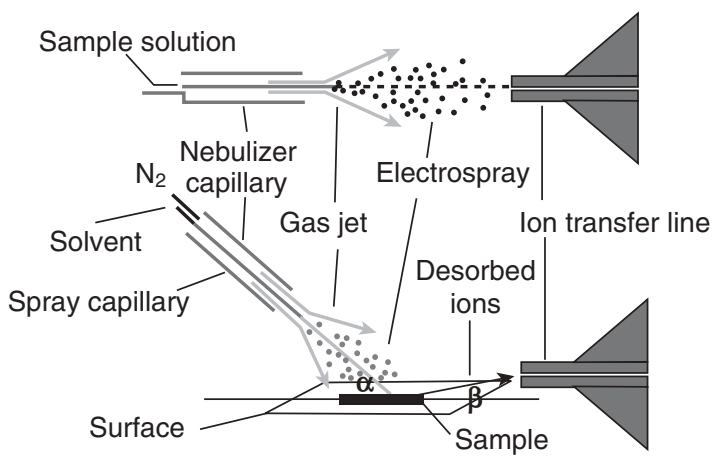

FIGURE 46.12 Schematic of the (A) ESSI and (B) DESI experiments.

Figure 46.11 contains the LDI mass spectra of PE500. The inset of Figure 46.11 highlights the isotope distribution of a silver-attached PE oligomer ion which matches that from the theory. The masses of the major peaks correspond to [alkane $+\mathrm{Ag}]^{+}$. Minor peaks, labeled $\mathrm{X}$, with masses matched with silver attached alkene, are also observed.

\subsubsection{DESI and ESSI}

Two complementary techniques, ESSI and DESI are introduced as variants of ESI to measure average molecular weights and molecular weight distributions of solution-phase and solid-phase polymer samples, respectively. For both techniques, high flow rates of nebulizing gas produce small initial droplets, and thus, facilitate solvent evaporation. The schematic diagram for ESSI and DESI sources is given in Figure 46.12 [12].

The following protocol describes application of DESI and ESSI sources in analysis of industrial polymers [12].

\section{Material}

Poly(ethylene glycol) $\left(\mathrm{M}_{\mathrm{w}}=3000\right)$

\section{Procedure}

1. Prepare the mass spectrometer according to the manufacturer's specifications and general procedures.

2. Analyze the sample using the operating conditions of the DESI and ESSI summarized in Table 46.2.

The positive ion DESI and ESSI mass spectra of the PEG sample are shown in Figure 46.13. Five distinct charge state envelopes with Gaussian distributions corresponding to multiply charged molecular ions $+2,+3$, $+4,+5$, and +6 are observed. Generally, for each charge state, more than one ion series is observed, for example, $[\mathrm{M}+2 \mathrm{Na}]^{2+},[\mathrm{M}+\mathrm{Na}+\mathrm{K}]^{2+}$, and so on. Polymeric molecules are ionized by binding to one or more adventitious ions $\mathrm{Na}^{+}, \mathrm{K}^{+}, \mathrm{NH}_{4}^{+}$, and $\mathrm{H}^{+}$. In the DESI mass spectrum $\mathrm{Na}^{+}$adducts predominate, whereas $\mathrm{NH}_{4}^{+}$ adducts are the most abundant in the ESSI spectra. Calculated molecular weight values $\left(\mathrm{M}_{\mathrm{w}}=3146\right.$ and 3209 from DESI and ESSI, respectively) are in good agreement with the value of 3000 reported by the manufacturer.

Critical Parameters The limitations of ESI discussed in Section 46.2.2.1 are also experienced with DESI and ESSI. The reliability of measured molecular weights and molecular weight distributions, especially for polydispersed polymers, is also affected by mass discrimination, changes in the cone potential in the atmospheric interface, and accommodation of multiple charges by large molecules. Loss of resolution and an increase in baseline may occur with the increase in number of possible types 
(A)

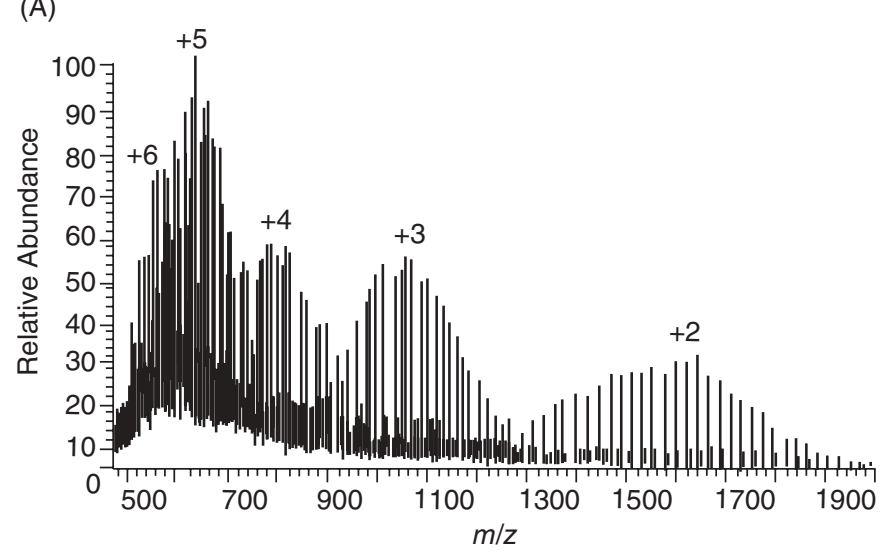

(B)

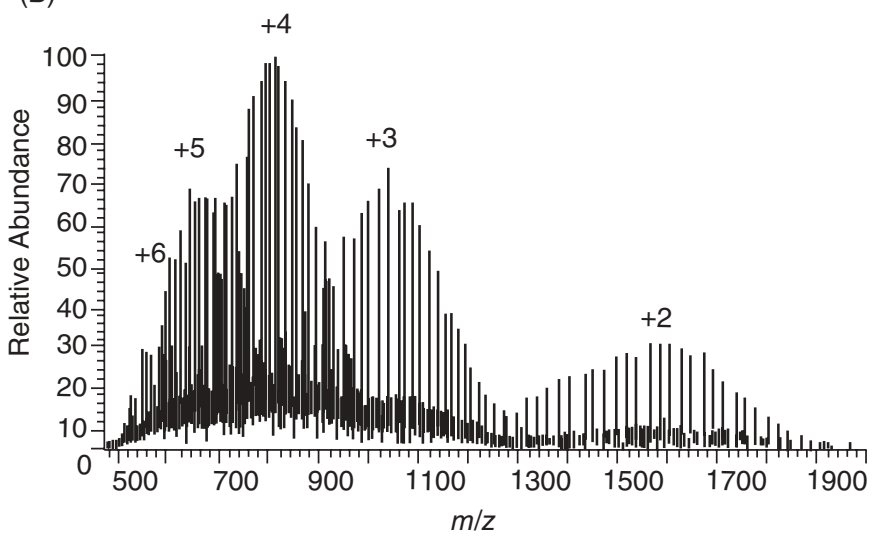

FIGURE 46.13 Mass spectra of PEG $\left(\mathrm{M}_{\mathrm{w}}=3000\right)$. (A) Positive ion DESI mass spectrum, $0.2 \mu \mathrm{g}$ per sample spot, spray solvent methanol:water, 1:1; (B) positive ion ESSI mass spectrum, sample concentration $55 \mu \mathrm{g} / \mathrm{mL}$ in methanol:water, $1: 1$.

TABLE 46.2 DESI and ESSI Source Settings

\begin{tabular}{|c|c|c|}
\hline & DESI & ESSI \\
\hline Electrospray voltage $^{a}$ & $5 \mathrm{kV}$ & $5 \mathrm{kV}$ \\
\hline Electrospray flow rate & $5 \mu \mathrm{L} / \mathrm{min}$ & $5 \mu \mathrm{L} / \mathrm{min}$ \\
\hline Incident/collection angle & 50/10 degrees & N/A \\
\hline MS inlet-sample distance & $10 \mathrm{~mm}$ & N/A \\
\hline Tip-sample distance & $5 \mathrm{~mm}$ & $\mathrm{~N} / \mathrm{A}$ \\
\hline Nebulizing gas pressure ${ }^{b}$ & 200 psi & 200 psi \\
\hline Tip-capillary inlet distance & N/A & $30 \mathrm{~mm}$ \\
\hline Type of surface & Paper & $\mathrm{N} / \mathrm{A}$ \\
\hline Liquid carrier & Methanol:water, 1:1 & \\
\hline Sample concentration & $0.2 \mu \mathrm{g}$ & $55 \mu \mathrm{g} / \mathrm{mL}$ \\
\hline Mass spectrometer & $\begin{array}{l}\text { Ion trap } m / z \text { range } \\
2000 \mathrm{Da}\end{array}$ & \\
\hline
\end{tabular}

${ }^{a}$ Applied to the syringe needle.

${ }^{b}$ Measured at the nitrogen gas tank.

of ionic clusters for charges +4 and higher. The choice of solvent is of critical importance, especially for hydrophobic polymers, as tendency toward multiple charging, and thus, accessible mass range, decreases in low polarity solvents. Thus, dissolution systems, usually mixtures of polar and nonpolar solvents needed for hydrophobic polymers, may produce no discrimination among different molecular weight oligomers.

The sensitivity of both DESI and ESSI increases with increasing ionization efficiency and decreases with an increase in number of ion series generated.

\subsection{POLYMER DEGRADATION}

\subsubsection{Photochemical and Oxidative}

By the application of MALDI-MS, precise information on the size, structure, and end groups of oligomers origi- nated in the oxidation process is obtained. Thus, the oxidation processes occurring in complex macromolecular systems can also be understood. In general, the soluble part of the thermo- or photooxidized polymer samples are analyzed according to the classical procedures for MALDI analysis. At least two different films should be analyzed at each exposure time. The structural identification of MALDI peaks are mainly made on the basis of empirical formulas. Isotopic resolution may help considerably in the peak assignment process through the comparison of the relative intensities of isotopic peaks corresponding to oligomers of increasing molar mass.

\subsubsection{Thermal Degradation}

The knowledge of the thermal degradation characteristics of polymers such as thermal stability, degradation products, and mechanism has significant importance for both academic and industrial points of view, especially for developing a rational technology of polymer processing, high temperature applications, and recycling processes. The thermal characterization techniques that involve a mass spectrometer as a detection system have the advantage of higher sensitivity, reproducibility, minimal sample preparation, and consumption and speed of analysis. MS has been interfaced to several thermal analysis methods such as thermogravimetry-differential thermal analysismass spectrometry (TG-DTA-MS), thermogravimetrydifferential thermogravimetry-mass spectrometry (TGDTG-MS), thermogravimetry-differential thermal analysis/ differential scanning calorimetry-mass spectrometry (TG-DTA/DSC-MS), simultaneous thermal analysismass spectrometry (STA-MS) and thermogravimetricdifferential thermogravimetric-differential thermal 
analysis-mass spectrometry (TG-DTG-DTA-MS). Furthermore, direct pyrolysis, DP-MS, and py-(GC-MS) techniques are also applied to investigate thermal degradation characteristics of macromolecules. Quadrupole mass spectrometers, QMS, are most widely coupled to thermogravimetry (TG) and gas chromatography (GC) because of their relatively small size, reasonable cost, sufficiently rapid scan rate, and high tolerance for pressure.

46.4.2.1 TG-MS TG-MS enables, in addition to the weight loss information, identification of the evolved gases in sequential order during thermal or thermooxidative degradation of a polymer in controlled atmospheric conditions $[13,14]$. In addition, the technique is also used to differentiate trapped solvents, unreacted reagents, and trace impurities. As the products of degradation are flushed out with the purged gas, the possibility of secondary reactions is reduced compared to sealed tube pyrolysis experiments. No sample contamination occurs and sample preparation is minimal.

The following protocol describes a procedure to investigate the effect of humidity on thermal decomposition of terephthalate polyester. The same procedure can be applied to any TG-MS analyses [13].

\section{Material}

Poly(butylene terephthalate), PBT

\section{Procedure}

1. Prepare the MS for analysis according to the general procedure.

2. Analyze the sample using the operating conditions of the TG-MS system summarized in Table 46.3.

Figure 46.14 shows results of TG-MS in dry $\mathrm{He}$ flow. The simultaneous total ion current (TIC) curve (variation of total ion yield as a function of temperature) is in good agreement with the TG curve.

Although the mass spectrum appears to be complex due to further dissociation during electron impact (EI) ionization of various evolved gases, comparison of the mass spectra with the National Institute of Standards and Technology (NIST) database allows for the identification of butadiene $(\mathrm{m} / \mathrm{z} 27,39$, and 54 ions $)$, benzoic acid $(\mathrm{m} / \mathrm{z} 51,77,105$, and 122), and monoalkenyl and dialkenyl esters of terephthalic acid $(\mathrm{m} / \mathrm{z}, 149$ and 203, respectively).

3. Repeat the TG-MS experiment in helium atmosphere of controlled humidity (i.e., $\mathrm{P}_{\mathrm{H} 2 \mathrm{O}}=12 \mathrm{kPa}$ )

Note: The ion sensitivity of MS decreases by introducing large amount of water vapor.
Both the TG and TIC curves and the main decomposition products in helium atmosphere of controlled humidity are similar to those obtained in dry helium atmosphere (Figure 46.15). Presence of groups of new peaks at $65,121,149$, and $166 \mathrm{Da}$ and 31,42, 44, and 71 Da were attributed to formation of terephtalic acid and butylene glycol due to hydrolysis reactions in humid atmosphere.

Critical Parameters In thermal analysis, only volatile degradation products can be analyzed. In general, the analysis is complicated due to concurrent thermal degradation processes and further dissociation during ionization in the mass spectrometer. The most important variables affecting resolution are sample size, heating/ cooling rate, purge gas composition, and flow rate. The evolved gases should be transferred from TG to MS rapidly. High gas flow rate and low sample mass are needed to eliminate secondary reactions. Yet, as thermal degradation occurs in a close container, secondary reactions cannot be totally eliminated. The carrier gas strongly dilutes the products to be measured. Memory effects caused by either traces of previous samples should be avoided.

46.4.2.2 $\boldsymbol{P} \boldsymbol{y}$-MS Pyrolysis is the thermal degradation of a compound in an inert atmosphere or vacuum. The two py-MS techniques are py-(GC-MS), in which the complex thermal degradation products are separated by the gas chromatograph prior to analysis by the mass spectrometer, and DP-MS technique, in which thermal degradation occurs inside the mass spectrometer close to the ionization source.

py-(GC-MS) Py-(GC-MS) analysis involves identification of volatile products generated during thermal degradation in a pyrolyzer and separated by the GC $[15,16]$. Various pyrolyzers such as filament, Curie-point,

TABLE 46.3 Operating Conditions of TG-MS System

\begin{tabular}{ll}
\hline Carrier gas & $\mathrm{N}_{2} / \mathrm{He}(99.99 \%)$ \\
Heating rate & $10^{\circ} \mathrm{C} / \mathrm{min}$ \\
Temperature range & Room to $800^{\circ} \mathrm{C}$ \\
Transfer line & $\begin{array}{c}\text { Stainless steel capillary with } \\
\text { inactivated internal surface at } 280^{\circ} \mathrm{C}\end{array}$ \\
Sample & $2-10 \mathrm{mg}$ \\
Sample holder & Platinum crucible of $5 \mathrm{~mm}$ diameter \\
& and $2.5 \mathrm{~mm}$ height \\
Flow rate & $200 \mathrm{~cm}^{3} / \mathrm{min}$ \\
Ionization source & $70 \mathrm{eV} \mathrm{EI}$ \\
Mass spectrometer & Quadrupole \\
Mass range & $10-350 \mathrm{Da}$ \\
MS scan rate & $0.5 \mathrm{~s} /$ full scale \\
\hline
\end{tabular}


(A)

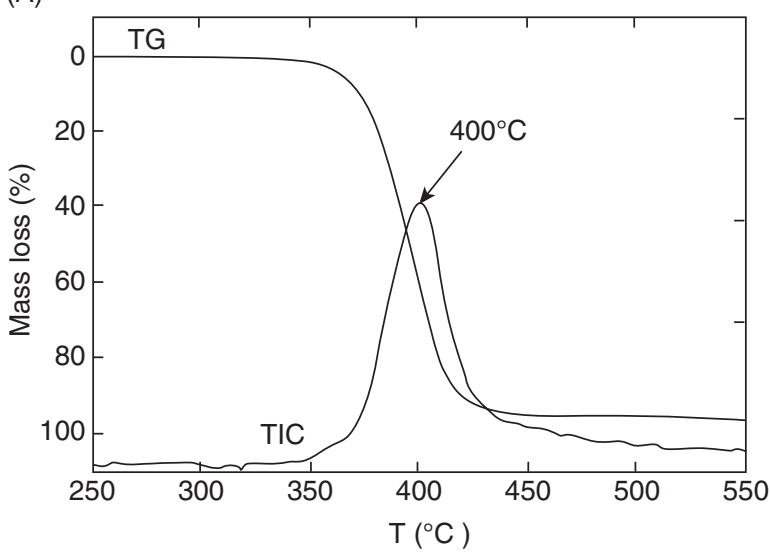

(B)

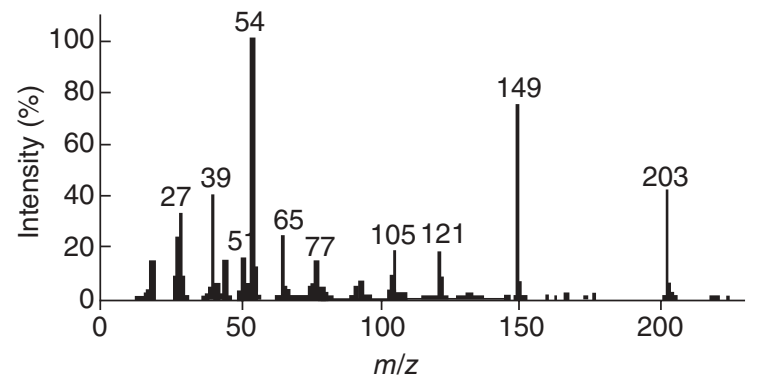

(C)

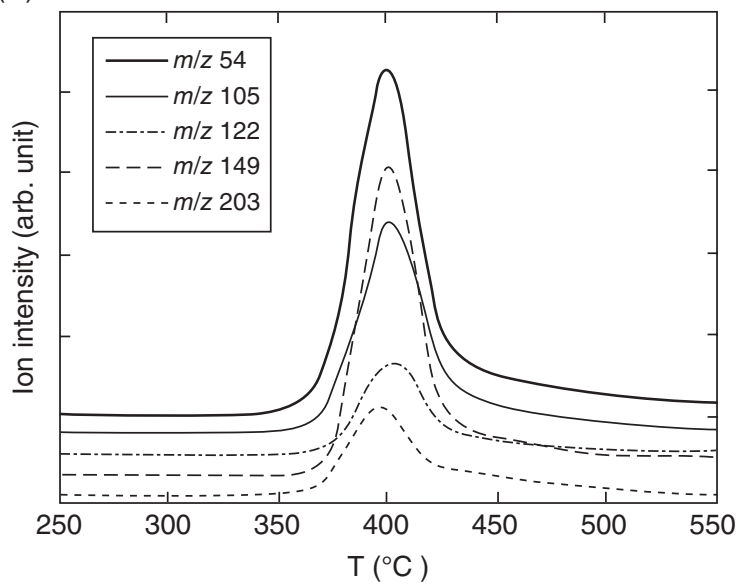

FIGURE 46.14 Results of TG-MS for PBT in dry helium flow at $10^{\circ} \mathrm{C} / \mathrm{min}$ : (A) TG and TIC curves, (B) mass spectrum for the evolved gas at $400^{\circ} \mathrm{C}$, and (C) mass chromatograms of $m / z, 54,105,122,149$, and 203. arb. unit, arbitrary units.

micro furnace, and laser heating pyrolyzers are commercially available. All volatile degradation products, under the flow of the carrier gas $\mathrm{N}_{2}$ or $\mathrm{He}$, not retained in the pyrolytical zone, injection system, or capillary column, are fed into the ion source of the mass spectrometer.

The QMS with the standard EI or chemical ionization (CI) or atmospheric ionization (API) source is the most
(A)

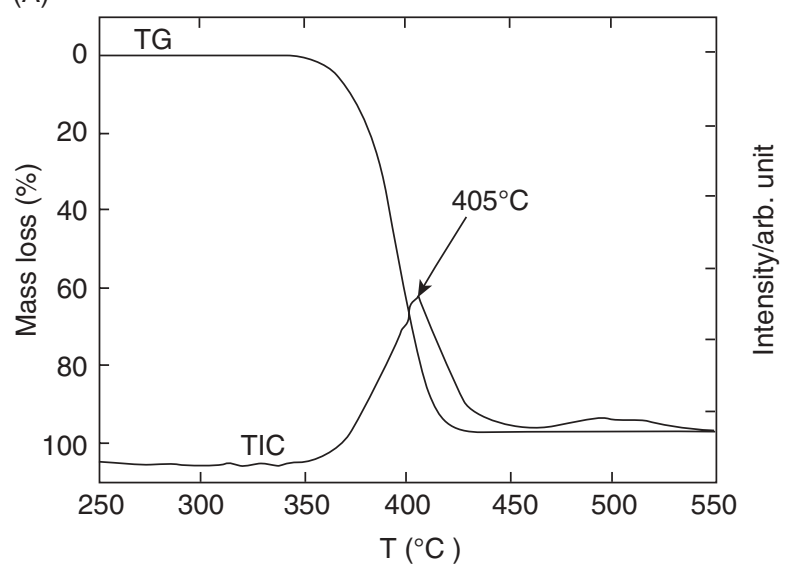

(B)

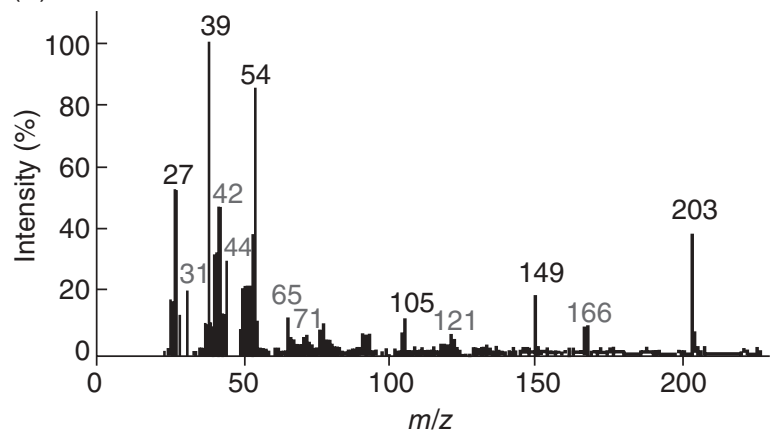

FIGURE 46.15 Results of TG-MS for PBT in helium atmosphere of controlled humidity $\left(\mathrm{P}_{\mathrm{H} 2 \mathrm{O}}=12 \mathrm{kPa}\right)$ at $10^{\circ} \mathrm{C} / \mathrm{min}$. (A) TG and TIC curves; (B) mass spectrum for the evolved gas at $405^{\circ} \mathrm{C}$.

commonly used mass spectrometer in py-(GC-MS) analysis. The EI yields more fragmentation useful for structural information and the EI spectra can be searched against standard libraries for identification while $\mathrm{CI}$ and API spectra provide molecular weight information. Each individual peak components of the specific pyrogram can be identified based on their mass spectra.

The following protocol describes a procedure for rapid characterization of synthetic polymers by heated filament and laser pyrolysis py-(GC-MS) [16].

\section{Materials}

Poly(methyl methacrylate) $\left(\mathrm{M}_{\mathrm{w}}=93,000 \mathrm{~g} / \mathrm{mol}\right)$

Nanocomposites: poly(methylmethacrylate) (PMMA) $-0,5,10,15$ and $20 \mathrm{wt} \%$ nanometric titanium oxide $\mathrm{TiO}_{2}$.

\section{Procedure}

1. Insert a fused-silica capillary column in the GC injector and condition the column at a temperature of $300^{\circ} \mathrm{C}$ according to the manufacturer's specifications. 
2. Connect the column to the MS and prepare the instrument for analysis.

3. Run a column blank using the operating conditions given in Table 46.4 .

Note: No peaks should be present in the gas chromatography-mass spectrometry (GC-MS) chromatogram. If some peaks are present, the source must be identified and eliminated (see critical parameters for guidelines).

4. Analyze the sample using operating conditions given in Table 46.4.

Figure 46.16 contains the TIC chromatograms of the pyrolyzates at $400^{\circ} \mathrm{C}$ for pure PMMA and its nanocomposites. The major decomposition product is MMA monomer and the addition of $\mathrm{TiO}_{2}$ does not change the nature of decomposition products. However, as the weight $\%$ of $\mathrm{TiO}_{2}$ increases yield of methanol $(\mathrm{m} / \mathrm{z}=32)$, propanoic acid methyl ester $(\mathrm{m} / \mathrm{z}=88)$ and methacrylic acid $(m / z=86)$ also increase (Figure 46.17). The emitted quantities are lower when PMMA is loaded with micrometric particles rather than with nanoparticles, except for propanoic acid methyl ester. This behavior can be related to the precision of measurements as propanoic acid methyl ester is produced in much lower amounts than methanol or methacrylic acid.
TABLE 46.4 Operating Conditions for Pyrolysis GC-MS

\begin{tabular}{ll}
\hline Instrument & \multicolumn{1}{c}{ GC-MS System } \\
\hline Pyrolyzer & CDS Pyroprobe 2000 (CDS \\
& Analytical Inc., Oxford, PA) \\
Mode of pyrolysis & Flash pyrolysis \\
Initial pyrolysis temperature & $25^{\circ} \mathrm{C}$ \\
Final pyrolysis temperature & $400^{\circ} \mathrm{C}$ \\
Heating rate & $5000^{\circ} \mathrm{C} / \mathrm{s}$ \\
Time at final temperature & $5 \mathrm{~s}$ \\
GC & \\
Carrier gas & Helium \\
Gas flow rate & $1.1 \mathrm{~mL} /$ min \\
Column & $\mathrm{Cross}-$ linked $5 \%$ PH-ME \\
& siloxane, 30 m length, \\
& 0.25 mm diameter, \\
& $0.25 \mu \mathrm{m}$ film thickness \\
Injector & or equivalent \\
& Split/splitless inlet with an \\
MS spectrometer & electronic pressure control, \\
Mass range & split ratio range: $1: 20-1: 50$ \\
Ionization source & Quadrupole \\
Source temperature & $10-750$ \\
Quadrupole temperature & $250^{\circ} \mathrm{C}$ \\
Interface temperature & $280^{\circ} \mathrm{C}$ \\
Sample & $1 \mathrm{mg}$ \\
\hline
\end{tabular}
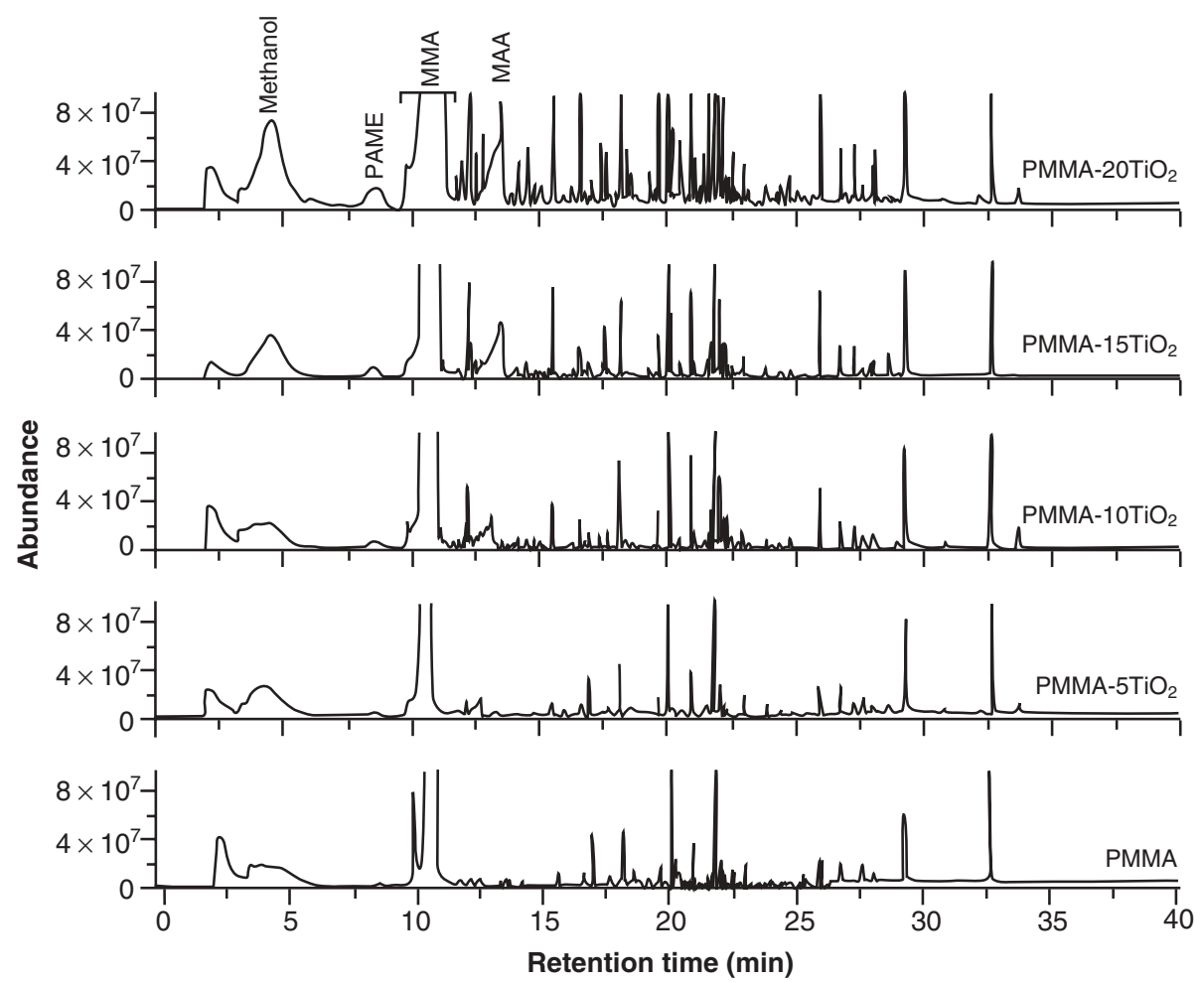

FIGURE 46.16 TIC of the pyrolysis products at $400^{\circ} \mathrm{C}$ of pure PMMA and its nanocomposites. 


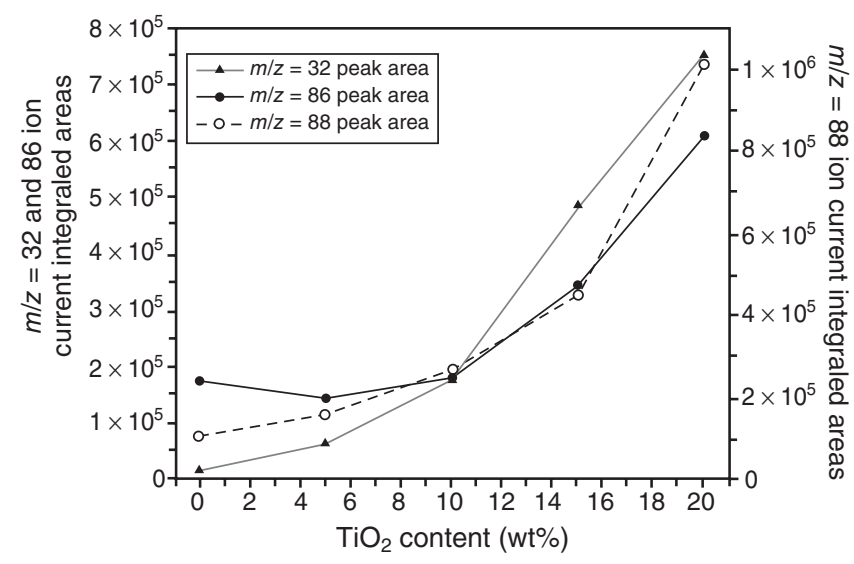

FIGURE 46.17 Ion current integrated areas for $\mathrm{m} / \mathrm{z}=32$ (methanol), $m / z=86$ (methacrylic acid), and $m / z=88$ (propanoic acid methyl ester).

Critical Parameters With the application of py-(GCMS), only stable volatile thermal degradation products can be identified. As thermal degradation occurs in a close container, secondary reactions cannot be eliminated totally.

Heating should be instantaneous to prevent drawn out transfer of the pyrolyzates through the injection port. Heated filament and Curie-point pyrolysis result in less secondary pyrolysis products compared to furnace pyrolysis. Curie-point pyrolyzers accurately reproduce pyrolysis conditions with a rapid temperature rise time, yet the choice of different pyrolysis temperatures is limited. Very little sample preparation or pretreatment is required for laser pyrolysis; however, a specific laser wavelength may not be appropriate for all types of samples.

As pyrolyzers are mounted external to the GC system, deposition of higher boiling point pyrolyzates and condensation of thermal degradation products in the transfer line is likely causing discrimination of high mass components and sample losses.

The transfer of pyrolyzates to the GC column should be rapid and efficient. To reduce the volume and the surface area the pyrolyzates travel, the interfacing between the pyrolyzer and the GC injection port should be kept minimal in order to ensure that all volatiles are fed into the GC column. The interface should be kept hot and insulated to prevent condensation reactions and presence of cold spots.

The sample size must be small and homogenous so that all of it degrades rapidly and column capacity is not exceeded. There is always the possibility of not detecting some of the thermal degradation products retained in the pyrolytical zone, injection system, or capillary column as a consequence of molecular weight and high polarity. Polar pyrolyzates, even if they enter the GC column, may often display peak tailing characteristics, poor reproducibility, long elution times, and in some cases no chromatographic peak. In situ derivatization of polar species into nonpolar volatile compounds reduces this problem to some extend.

Presence of peaks in the column blank indicates contamination of the carrier gas or column, or rarely, gas lines. The source of contamination should be examined and replaced, if necessary.

46.4.2.3 Direct py-MS DP-MS technique is the only technique in which secondary and condensation reactions are avoided and detection of high mass pyrolyzates and unstable thermal degradation products is possible. Thus, a better understanding of the thermal characteristics, polymerization, cross-linking, and char formation processes can be achieved. However, direct pyrolysis mass spectra of polymers are almost always very complicated due to concurrent degradation processes and dissociative ionization of the thermal degradation products inside the mass spectrometer. Therefore, soft ionization techniques are preferential.

The following protocol describes a procedure for investigation of thermal degradation characteristics of some homopolymers and copolymers by DP-MS.

\section{Materials}

PMMA

Poly(ethylene oxide), PEO

Poly(vinyl acetate), PVAc

Polystyrene-co-block(poly(4-vinyl pyridine)), PS-bP4VP

Poly(methylmethacrylate) nanowebs containing cyclodextrin and aniline, PMMA-CD-aniline

\section{Temperature Program}

Increase the temperature to $50^{\circ} \mathrm{C}$ in $1 \mathrm{~s}$ and keep the temperature constant at $50^{\circ} \mathrm{C}$ for $5 \mathrm{~min}$ to remove any absorbed water and then increase the temperature to $650^{\circ} \mathrm{C}$ (maximum attainable temperature) at a heating rate of $10^{\circ} \mathrm{C} / \mathrm{min}$, and keep constant for an additional $10 \mathrm{~min}$ at $650^{\circ} \mathrm{C}$.

\section{Procedure}

1. Prepare the MS instrument according to manufacturer's specifications

2. Place an empty quartz sample vial into the direct insertion probe and insert it into the mass spectrometer after pumping the connecting lines according to the manufacturer's specifications. 
3. When the pressure is dropped, take a background spectrum using the temperature program selected.

Note: No peaks other than background gases should be present in the background spectrum. If some extra peaks are present, check the system (see critical parameters).

4. Remove the direct insertion probe from the system and install about $10 \mu \mathrm{g}$ sample into the vial and insert the probe into the mass spectrometer as described above.

5. Start the analysis using the temperature program selected.

6. When the analysis is completed, cool the probe down to the limit given by the manufacturer. Remove the probe from the mass spectrometer.

\section{Data Analysis}

1. Examine the shape of the TIC curve (variation of total ion yield as a function of temperature) and record the maximum of the peaks present in the TIC curve.

Note: The presence of more than one peak in the TIC curve indicates either a multistep thermal degradation and/or presence of more than one component.

2. A single and relatively sharp peak in the TIC curve is usually an indication of a single-step thermal degradation mechanism. One possibility is depolymerization mechanism yielding mainly the monomer. In this case, the pyrolysis mass spectra are usually simple and resemble that of the monomer. Thus, comparison of the spectrum with the mass spectrum of the monomer is always necessary.

3. Analyze the mass spectrum recorded at the peak maximum. Try to identify peaks due to monomer or any diagnostic fragments. Compare the singleion evolution profiles of all intense and high mass ions and group the similar ones.

\section{Pyrolysis of PMMA}

Figure 46.18 contains the TIC curve and the pyrolysis mass spectra recorded at $398.6^{\circ} \mathrm{C}$ (the peak maximum) during the pyrolysis of PMMA. The pyrolysis mass spectrum is identical to that of the methyl methacrylate, indicating evolution of the monomer during thermal degradation. Thus, a depolymerization mechanism for thermal degradation can directly be assigned. Single-ion evolution profiles (single ion pyrograms) of MMA (100 Da), $\mathrm{CH}_{3} \mathrm{CO}$ (69 Da), and $\mathrm{CH}_{2} \mathrm{CCH}_{3}$ (41 Da) ions show similar trends, confirming one-step thermal degradation process.

\section{Pyrolysis of PEO}

The TIC curve recorded during the pyrolysis of PEO is shown in Figure 46.19. A single peak is present in the TIC curve. Yet, the mass spectrum recorded at $412^{\circ} \mathrm{C}$ is quite complex and does not resemble that of the monomer. Similarities in the single-ion evolution profiles suggest a single-step thermal degradation involving random cleavages along the polymer chain, yielding various fragments stabilized by $\mathrm{H}$-transfer reactions. Evolution profiles of $\mathrm{O}=\mathrm{CHCH}_{2}(43 \mathrm{Da}), \mathrm{C}_{5} \mathrm{H}_{9} \mathrm{O}_{3}$ (117 Da), $\mathrm{C}_{5} \mathrm{H}_{9} \mathrm{O}_{3}$, (309 Da), and $\mathrm{C}_{5} \mathrm{H}_{9} \mathrm{O}_{3}(529 \mathrm{Da})$ are also included in the figure.

\section{Pyrolysis of PVAc}

The TIC curve recorded during the pyrolysis of PVAc shows two peaks indicating a two-step thermal degradation mechanism (Figure 46.20). The mass spectra recorded at 359 and $456^{\circ} \mathrm{C}$ and the trends in the singleion evolution profiles suggest the first step to be the loss of side chains and evolution of acetic acid, and the second step to be the decomposition of the unsaturated polymer backbone generated.

\section{Pyrolysis of PS-b-P4VP}

The TIC curve and the mass spectra recorded at the maxima of the peaks present in the TIC curve during the pyrolysis of PS-b-P4VP are shown in Figure 46.21. It is clear that thermal degradation takes place in two distinct temperature ranges at around 331 and $446^{\circ} \mathrm{C}$. The most intense peaks in these temperature ranges are due to monomers of P4VP and PS, 4-vinyl pyridine (105 Da) and styrene (104 Da), respectively, indicating that depolymerization is the main thermal degradation mechanism for both components of the copolymer. The pyrolysis mass spectra and the trends in the single-ion evolution profiles (dimer and trimer of 4-vinyl pyridine with $\mathrm{m} / z$ values 210 and $315 \mathrm{Da}$ and dimer and trimer of styrene with $\mathrm{m} / z$ values 208 and $312 \mathrm{Da}$ ) clearly point out that both components decompose independently (Figure 46.21C).

\section{Pyrolysis of PMMA Nanowebs Containing Cyclodextrin-Aniline Inclusion Complexes}

Direct pyrolysis mass spectrometric analyses of PMMA nanofibers containing cyclodextrin-aniline complexes clearly show evolution of components according to their volatilities and thermal stabilities (Figure 46.22). The results clearly reveal the stability and sustained release of aniline in higher temperature ranges after the production of nanowebs. 


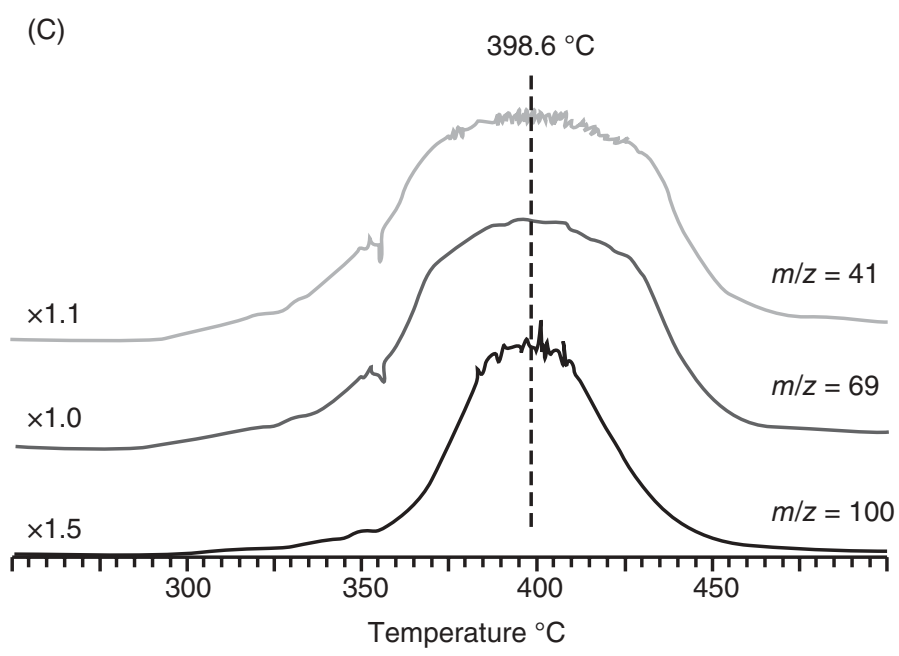

(B)
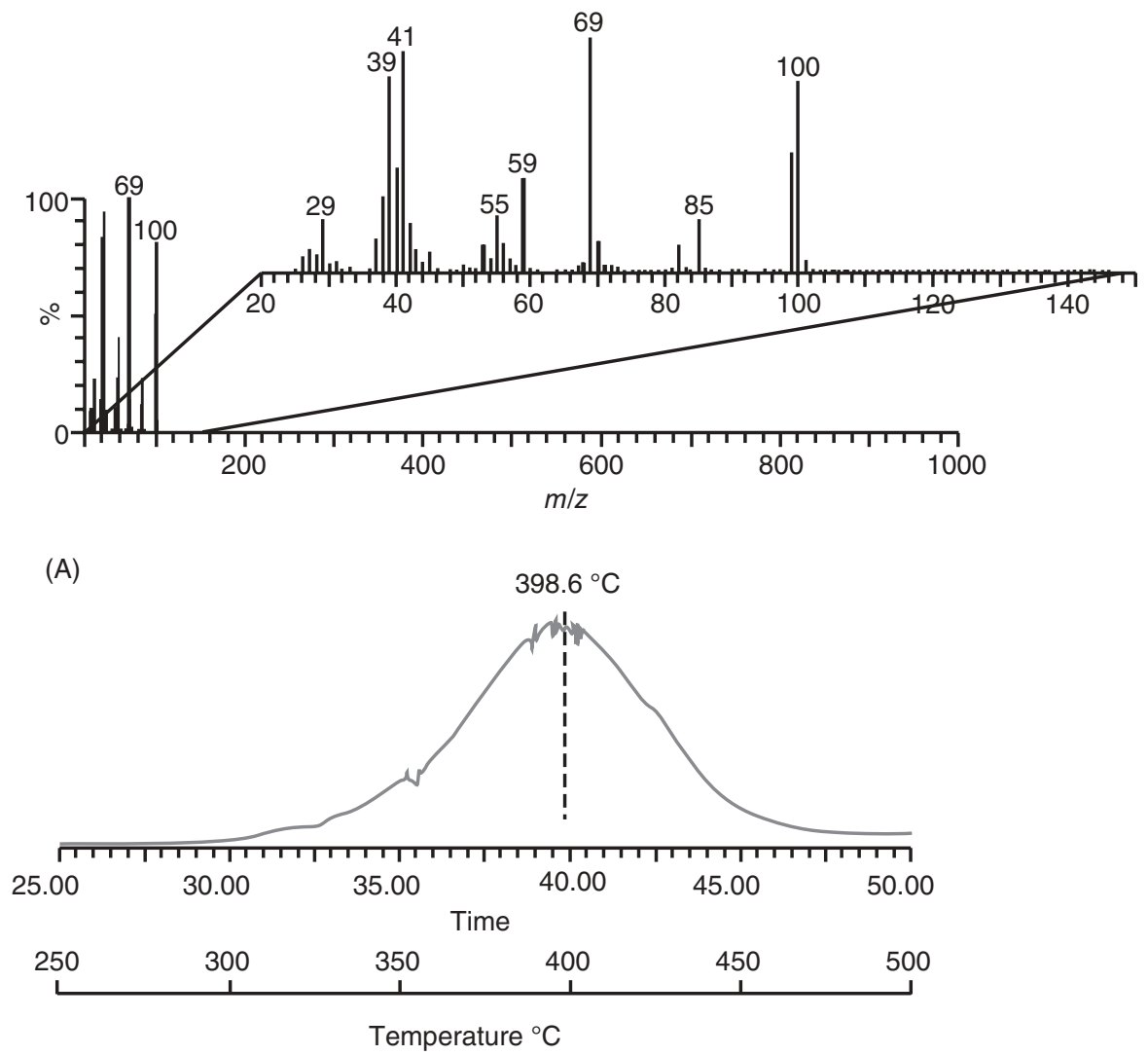

FIGURE 46.18 (A) TIC curve recorded during pyrolysis of PMMA. (B) The mass spectrum recorded at the peak maximum at $398.6^{\circ} \mathrm{C}$ (39.86 min). (C) Single ion evolution profiles of MMA (100 Da), $\mathrm{CH}_{3} \mathrm{CO}$ (69 Da), and $\mathrm{CH}_{2} \mathrm{CCH}_{3}(41 \mathrm{Da}$ ).

Critical Parameters Thermal degradation products further dissociate during ionization and yield very complicated pyrolysis mass spectra. Therefore, soft ionization techniques may seem to be more appropriate. However, for soft ionization techniques such as API and CI, secondary reactions may take place. Then, investigation of thermal degradation mechanism may be even more difficult. For applications that involve a CI source, the choice of reagent gas used has significant importance on the extent of fragmentation and ion molecule reactions. Thus, inert gases with moderate proton affinities such as Ar or $\mathrm{N}_{2}$ may be used to eliminate adduct formation reactions.

If an EI source is used, the analysis should also be repeated at low energies to minimize fragmentation and 
(C)

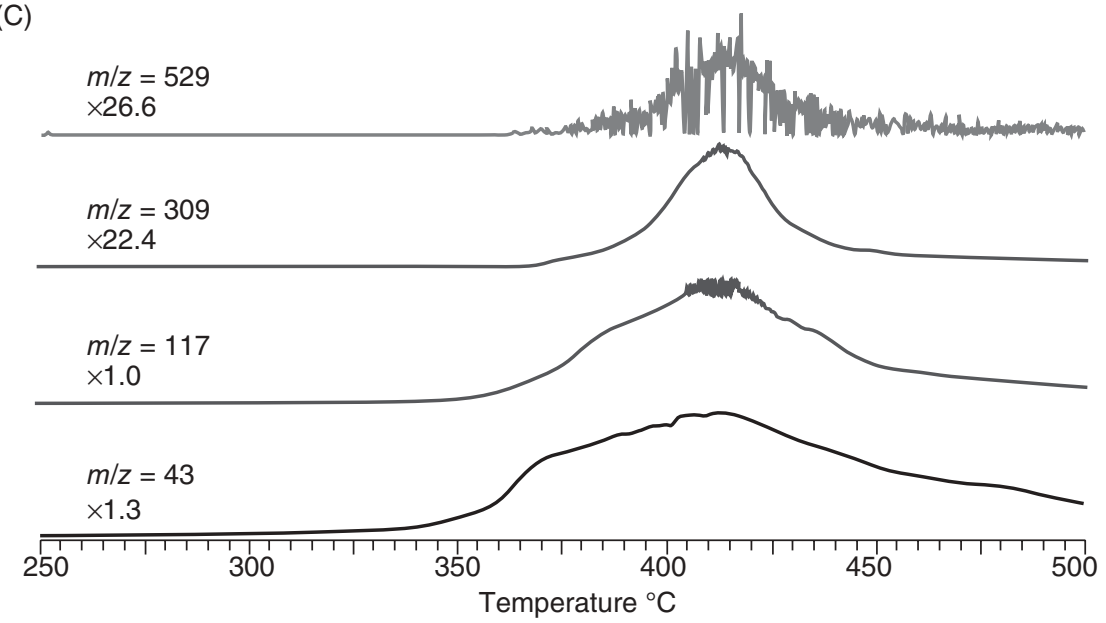

(B)
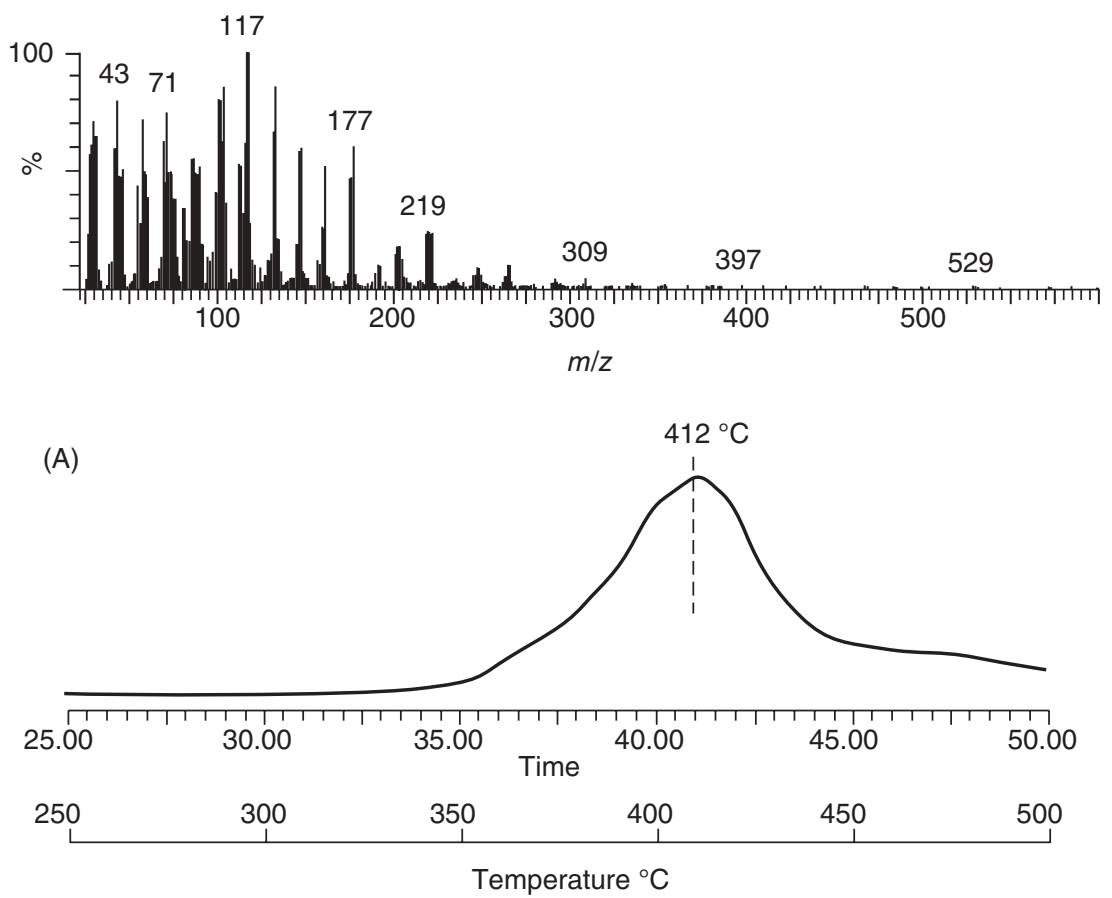

FIGURE 46.19 (A) TIC curve recorded during pyrolysis of PEO. (B) The mass spectrum recorded at the peak maximum at $398.6^{\circ} \mathrm{C}$ (39.86 min). (C) Single ion evolution profiles of $\mathrm{O}=\mathrm{CHCH}_{2}(43 \mathrm{Da}), \mathrm{C}_{5} \mathrm{H}_{9} \mathrm{O}_{3}(117 \mathrm{Da}), \mathrm{C}_{5} \mathrm{H}_{9} \mathrm{O}_{3}(309 \mathrm{Da}), \mathrm{and}_{5} \mathrm{H}_{9} \mathrm{O}_{3}$ (529 Da).

to differentiate the parent fragments generated during the thermal degradation.

Nevertheless, in py-MS analysis, it is not only the detection of a peak that is important, but also the variation of its intensity as a function of temperature, singleion evolution profiles, and single-ion pyrograms. All ions with identical evolution profiles should be grouped and analyzed separately. The fragment with the highest mass may be assumed to be generated during thermal degra- dation. On the other hand, the low mass fragments having similar evolution profiles may be generated either during thermal degradation or during ionization in the mass spectrometer.

Contamination is of high probability during pyrolysis studies of polymers. The presence of peaks other than the background gases in the background spectrum indicates contamination of the system. The system should be cleaned according to the manufacturer's specifications. 


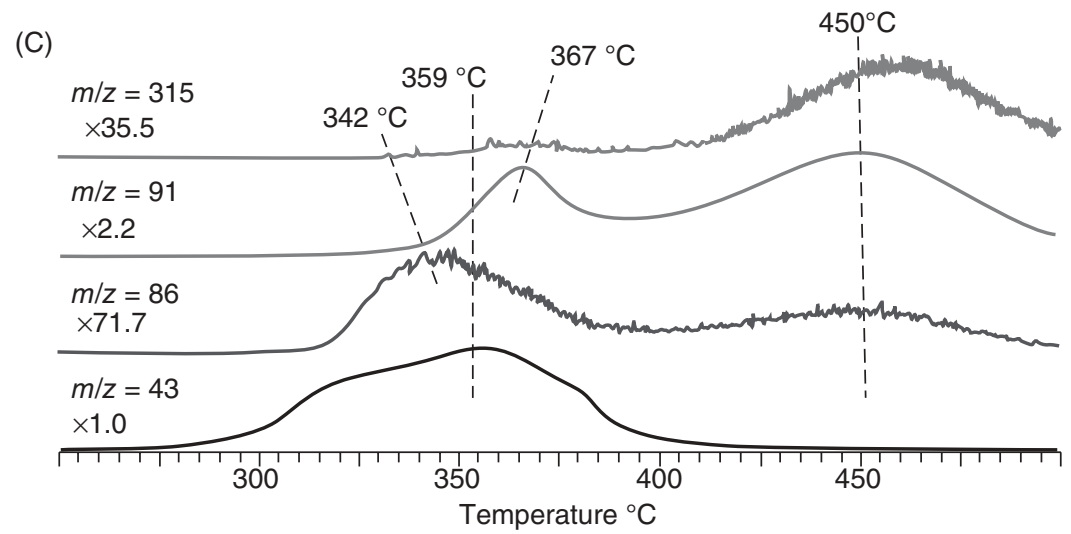

(B)
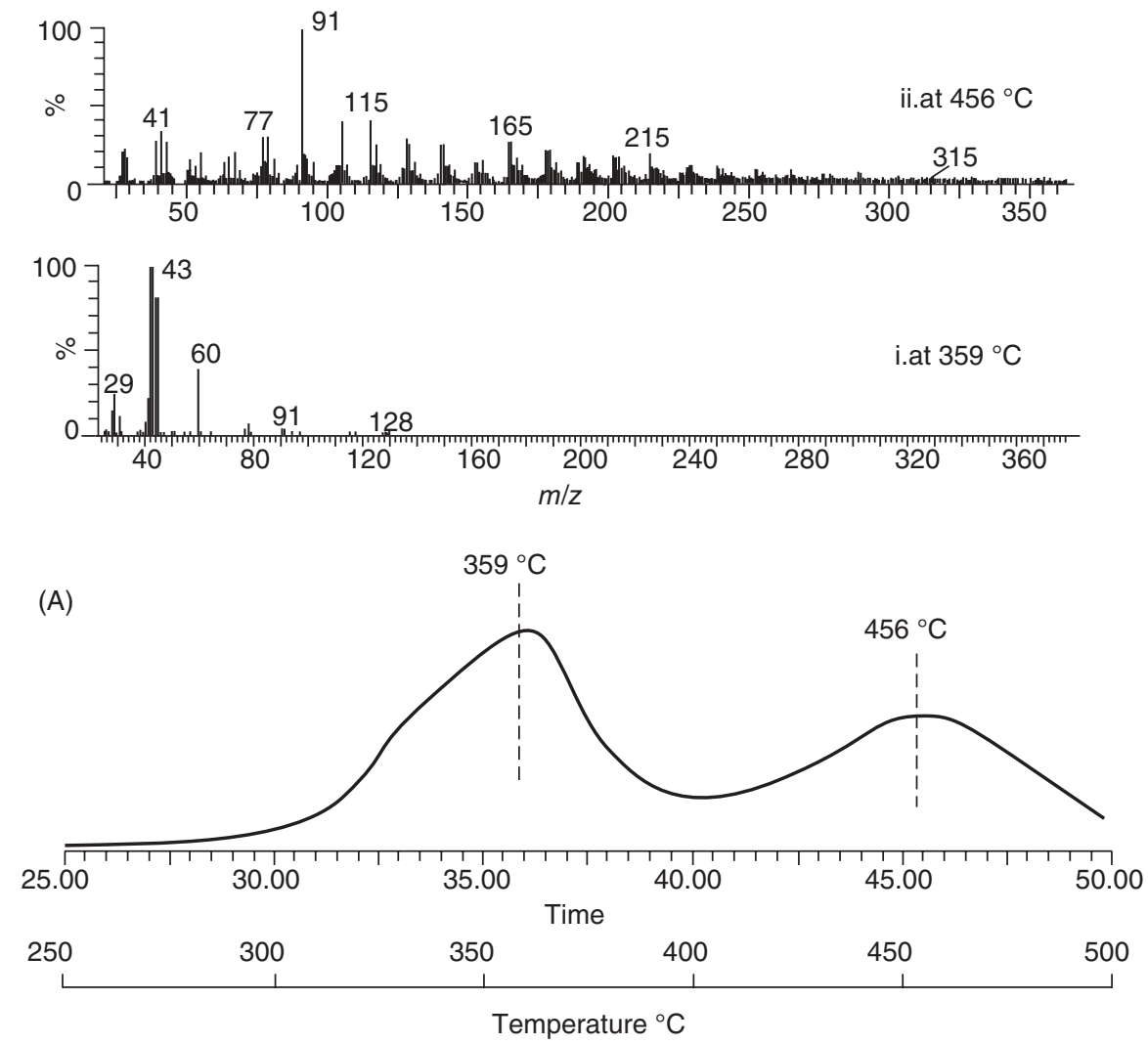

FIGURE 46.20 (A) TIC curve recorded during pyrolysis of PVAc. (B) The mass spectrum recorded at the peak maximum at 359 and $456^{\circ} \mathrm{C}$ and (C) single ion evolution profiles of $\mathrm{C}_{2} \mathrm{H}_{3} \mathrm{O}$, VAc, $\mathrm{C}_{7} \mathrm{H}_{7}$, and $\mathrm{C}_{24} \mathrm{H}_{2}$.

\subsection{POLYMER SURFACE AND INTERFACE}

TOF-SIMS is a unique tool for characterizing molecular surfaces with high lateral resolution. With this method, a focused beam of energetic ions is utilized to desorb the target molecules from a known spot on the sample. Static TOF-SIMS has shown to be the most useful technique for the evaluation of the conformations of a range of macromolecules on surfaces. The technique is based on a significant variation in the intensity of specific peaks between the related samples. Primary ions sources used are either ions of gaseous elements such as noble gases or ionized molecules such as $\mathrm{C}_{60}{ }^{+}$or $\mathrm{SF}_{5}{ }^{+}$or ions generated by surface ionization sources such as $\mathrm{Cs}+$ or $\mathrm{Ga}^{+}$.

The following protocol describes the investigation of the surface of a triblock copolymer, containing a solid-phase drug using $15 \mathrm{keV} \mathrm{Ga}^{+}$and $20 \mathrm{keV} \mathrm{C}_{60}{ }^{+}$ion beams [17]. 


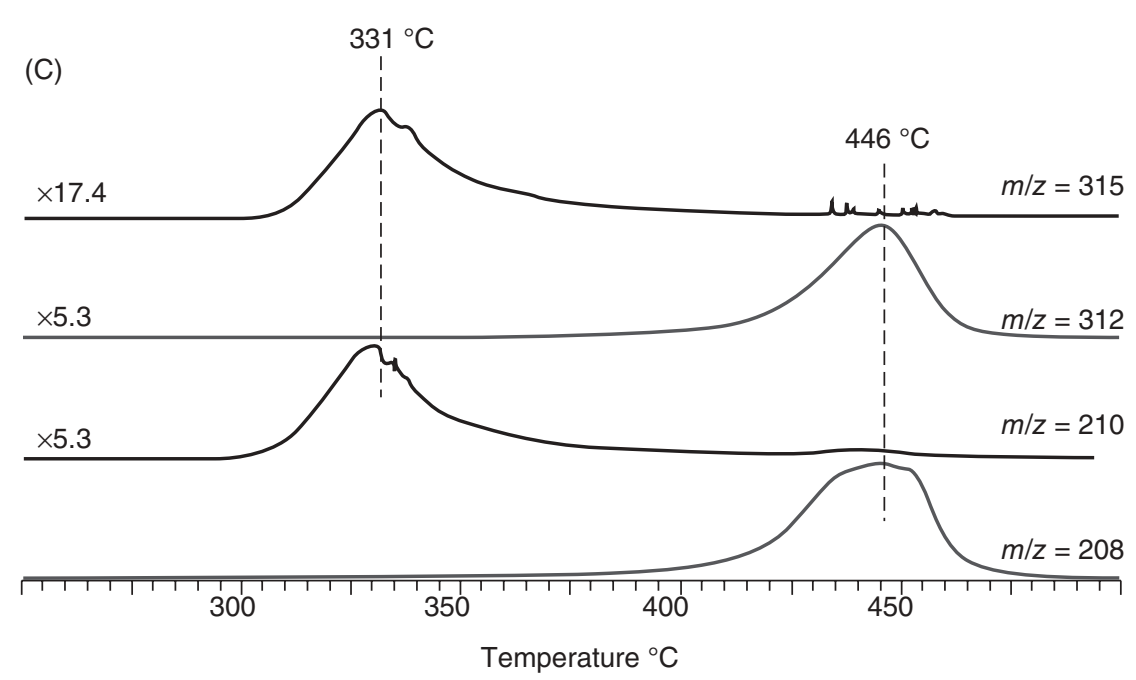

(B)
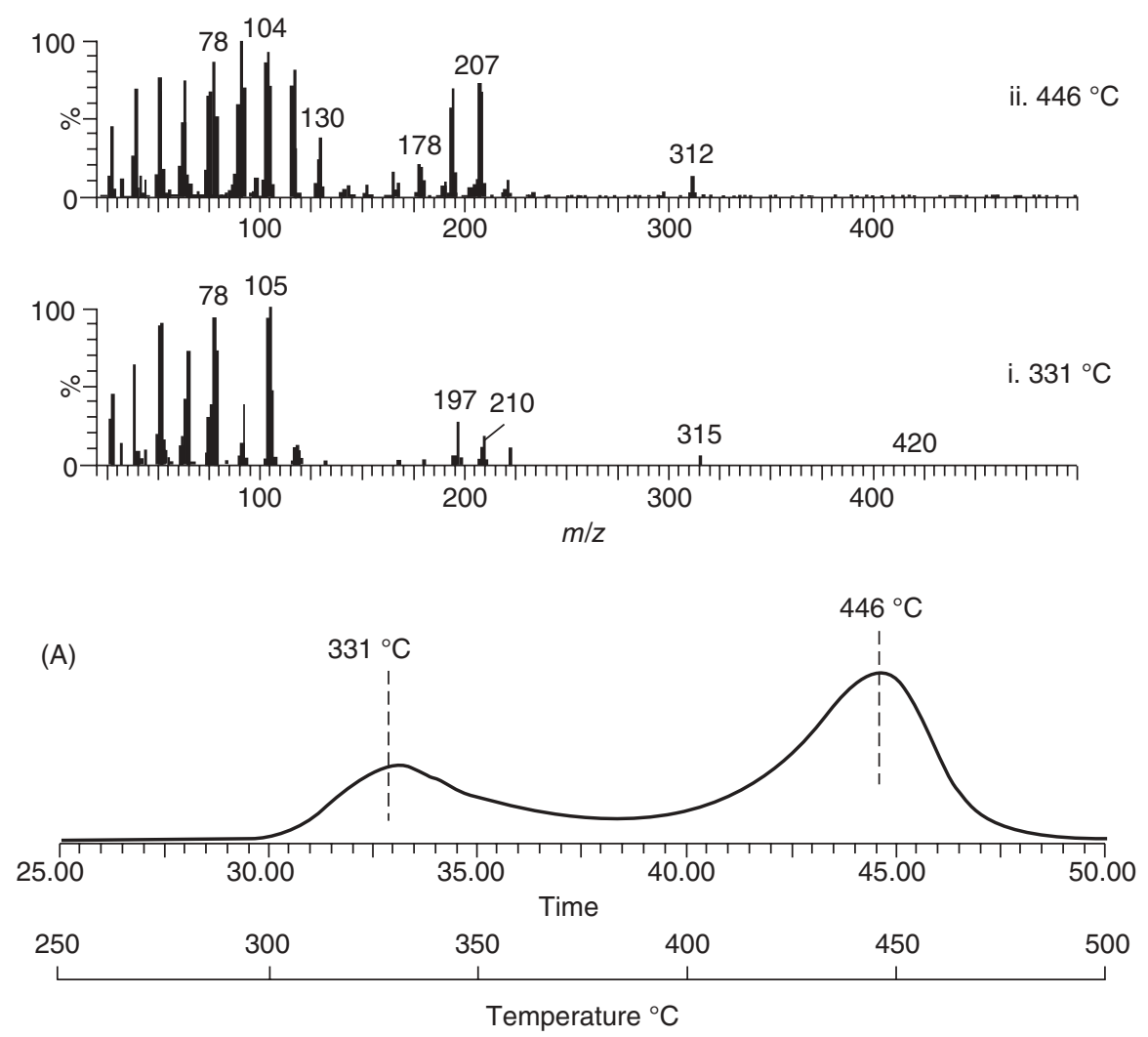

FIGURE 46.21 (A) TIC recorded during pyrolysis of PS-b-P4VP. (B) The mass spectrum recorded at the peak maxima at 331 and $446^{\circ} \mathrm{C}$ (39.86 min). (C) Single ion evolution profiles of some selected products. 


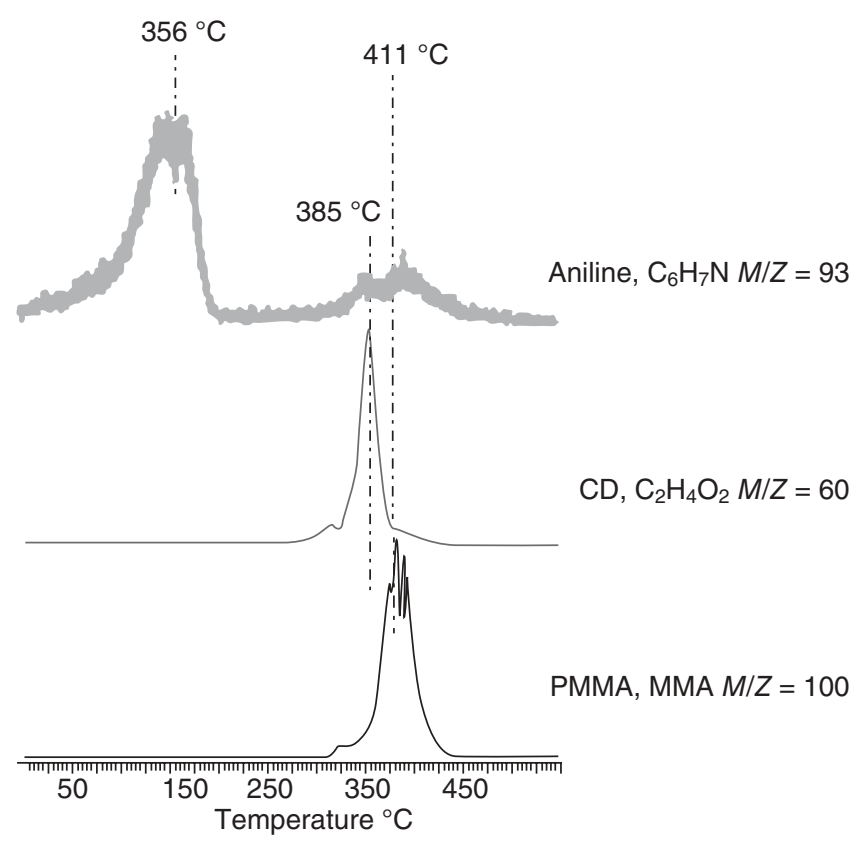

FIGURE 46.22 Single ion evolution profiles of MMA, $\mathrm{C}_{2} \mathrm{H}_{4} \mathrm{O}_{2}$ and aniline recorded during the pyrolysis of PMMA nanowebs containing cyclodextrin-aniline inclusion complexes.

\section{Materials}

Taxol(0.0 and $8.8 \mathrm{wt} \%)$ loaded poly(styrene- $b$-isobutylene$b$-styrene) (SIBS) cast from 75:25 toluene:THF solution on clean polyethylene terephthalate sheets.

\section{Procedure}

1. Prepare the mass spectrometer for analysis according to the manufacturer's specifications and general procedures.

2. Analyze the sample using the operational parameters given in Table 46.5 .

Positive, secondary ion mass spectra of SIBS polymer containing taxol recorded using $\mathrm{Ga}^{+}$ions and $\mathrm{C}_{60}{ }^{+}$ions under static conditions are shown in Figure 46.23. The $(\mathrm{M}+\mathrm{H})^{+}$ion from taxol is not observed while the contribution of polydimethyl siloxane (PDMS), which is innate to the polymer formulation, is quite significant with $\mathrm{Ga}^{+}$ions. The molecular ions of taxol and higher mass fragments are enhanced using $\mathrm{C}_{60}{ }^{+}$primary ions.

3. Obtain three-dimensional distribution of the species detected.

Figure 46.24 illustrates the intensity of secondary ion species from the polymer matrix plotted as a function of primary ion dose $\left(\mathrm{C}_{60}{ }^{+} / \mathrm{cm}^{2}\right)$. Peaks related to poly(styrene) and poly(isobutylene) fragments possess slightly lower intensity in the surface region and achieve
TABLE 46.5 Operational Parameters
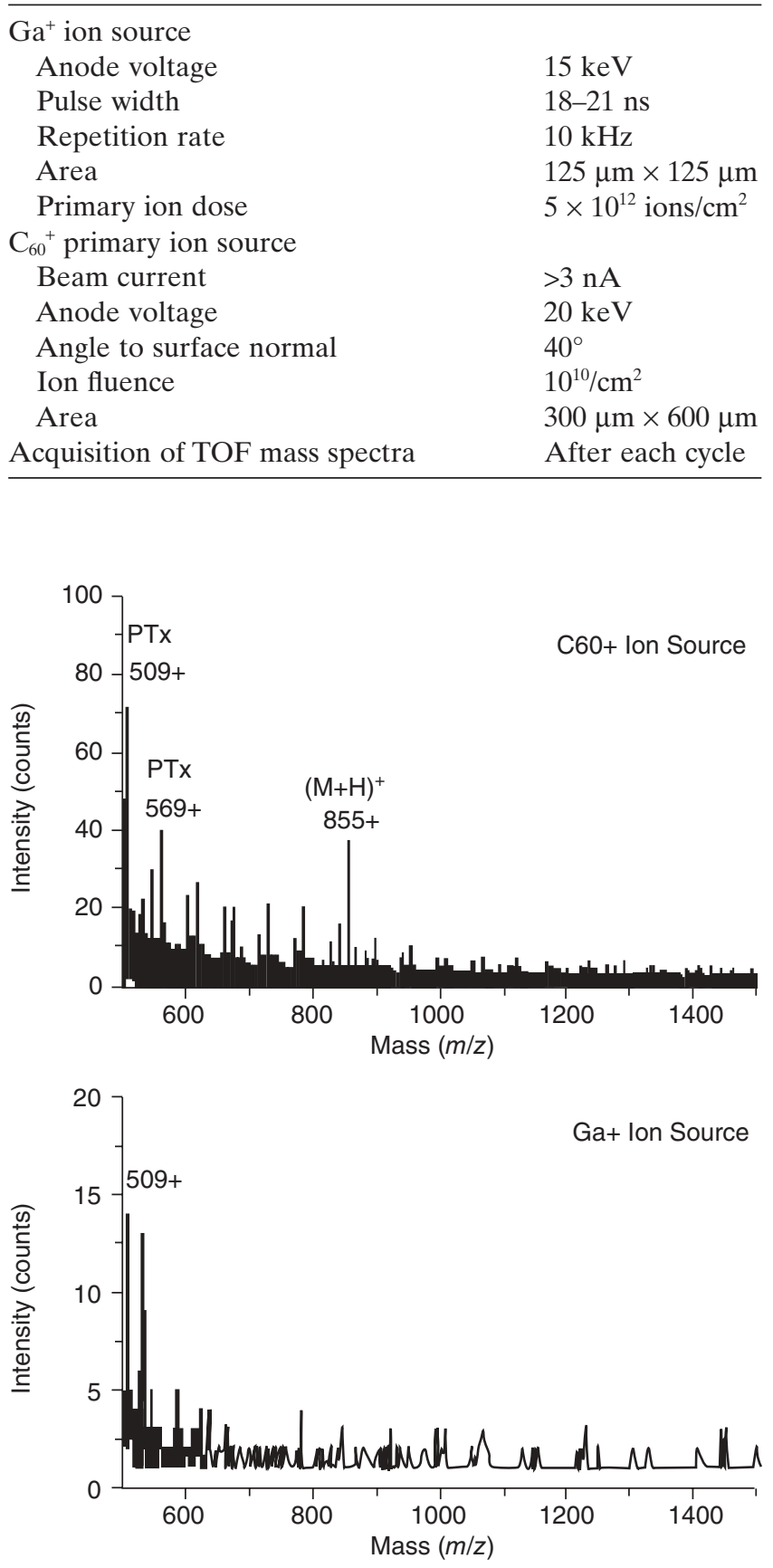

FIGURE 46.23 Positive, secondary ion mass spectra of SIBS polymer containing paclitaxel (PTx). Data were recorded using $\mathrm{Ga}^{+}$ions (lower spectrum) and $\mathrm{C}_{60}{ }^{+}$ions (upper spectrum) under static conditions.

a relatively uniform intensity profile (i.e., steady state) at higher ion doses. In contrast, the PDMS- and taxolrelated peaks possess higher intensity near the surface and approach a steady-state intensity after a primary ion dose of approximately $2 \times 10^{13} \mathrm{ions} / \mathrm{cm}^{2}$. 


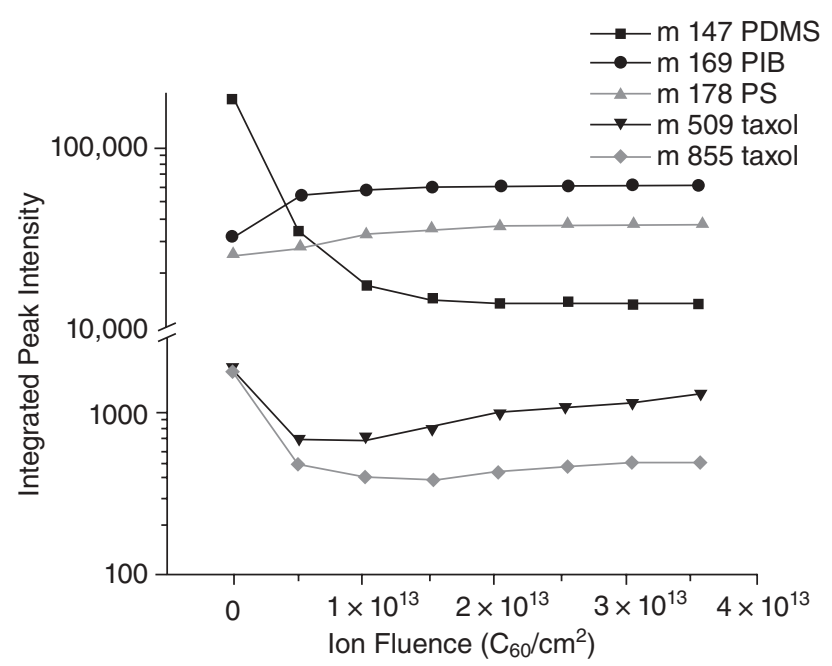

FIGURE 46.24 Depth profile of SIBS polymer containing PTx. Secondary ion intensities for select species plotted as a function of primary ion $\left(\mathrm{C}_{60}{ }^{+}\right)$dose.

Note: If a so-called matrix effect exists, ion yields will be altered as a function of sputter time and then all species should be affected and show similar intensity distributions.

Consequently, it is reasonable to assess that there is an enrichment of PDMS and taxol at the surface of the SIBS polymer, although, it is not possible to quantitate these findings.

Information about the three-dimensional distribution of species would be limited if the sample is significantly damaged during the bombardment process.

Critical Parameters There are two major difficulties that prevent development of this tool to its full capability. First, the ion beam induces significant fragmentation of molecules during desorption, which increases the congestion of the mass spectra and destroys the chemical nature of the sample itself. This latter effect is particularly noticeable if the dose of primary ions exceeds $\sim 1 \%$ of the number of surface molecules.

The number of molecules available for analysis becomes vanishingly small as the pixel size approaches 0 . For example, if there are $1,000,000$ molecules per square micrometer and $1 \%$ of these are sputtered, then with an ionization efficiency of $<1$ part in 104, only one molecule would be available for detection.

To enhance the secondary ion intensity, an essential indicator of the sensitivity, is the use of polyatomic primary projectiles such as $\mathrm{SF}_{5}{ }^{+}, \mathrm{Au}_{3}{ }^{+}, \mathrm{Bi}_{3}{ }^{+}$, and $\mathrm{C}_{60}{ }^{+}$.

\section{REFERENCES}

1. Weidner, S.M., Trimpin, S. (2008) Mass spectrometry of synthetic polymers. Anal. Chem., 80, 4349-4361.

2. Peacock, P.M., McEwen, C.N. (2006) Mass spectrometry of synthetic polymers. Anal. Chem., 78, 3957-2964.

3. Wetzel, S.J., Guttman, C.M., Flynn, K.M. (2006) Significant parameters in the optimization of MALDI-TOF-MS for synthetic polymers. J. Am. Soc. Mass Spectrom., 17, 246252.

4. Zhang, L.K., Rempel, D., Pramanik, B.N., Gross, M.L. (2005) Accurate mass measurements by Fourier transform mass spectrometry. Mass Spectrom. Rev., 24, 286-309.

5. El-Aneed, A., Cohen, A., Banoub, J. (2009) Mass spectrometry, review of the basics: electrospray, MALDI, and commonly used mass analyzers. Appl. Spectrosc. Rev., 44, 210-230.

6. Wu, K.J., Odom, R.W. (1998) Characterizing synthetic polymers by MALDI MS. Anal. Chem., 70, A456A461.

7. Ma, Z., Qiang, L.L., Fan, Q.L., Wang, Y.Y., Pu, K.Y., Yin, R., Huang, W. (2007) Direct laser desorption/ionization time-of-flight mass spectrometry of conjugated polymers. J. Mass Spectrom., 42, 20-24.

8. Mize, T.H., Simonsick, W.J., Amster, I.J. (2003) Characterization of polyesters by matrix-assisted laser desorption/ionization and Fourier transform mass spectrometry. Eur. J. Mass Spectrom., 9, 473-486.

9. Yalcin, T., Gabryelski, W., Li, L. (2000) Structural analysis of polymer end groups by electrospray ionization highenergy collision-induced dissociation tandem mass spectrometry. Anal. Chem., 72(16), 3847-3852.

10. van Leeuwen, S.M., Tan, B., Grijpma, D.W., Feijen, J., Karst, U. (2007) Characterization of the chemical composition of a block copolymer by liquid chromatography/ mass spectrometry using atmospheric pressure chemical ionization and electrospray ionization. Rapid Commun. Mass Spectrom., 21, 2629-2637.

11. Chen, R., Yalcin, T., Wallace, T.W.E., Guttman, C.M., Li, L. (2001) Laser desorption ionization and MALDI time-offlight mass spectrometry for low molecular mass polyethylene analysis. J. Am. Soc. Mass Spectrom., 12, 11861192.

12. Nefliu, M., Venter, A., Cooks, R.G. (2006) Desorption electrospray ionization and electrosonic spray ionization for solid- and solution-phase analysis of industrial polymers. Chem. Commun., 8, 888-890.

13. Arii, T., Masuda, Y. (2004) The effect of humidity on thermal decomposition of terephthalate polyester. J. Anal. Appl. Pyrol., 71, 525-536.

14. Raemaekers, K.G.H., Bart, J.C.J. (1997) Applications of simultaneous thermogravimetry-mass spectrometry in polymer analysis. Thermochim. Acta, 295, 1-58. 
15. Sobeih, K.L., Baron, M., Gonzalez-Rodriguez, J. (2008) Recent trends and developments in pyrolysis-gas chromatography. J. Chromatogr. A, 1186, 51-66.

16. Laachachi, A., Ferriol, M., Cochez, M., Ruchand, D., Lopez-Cuesta, J.M. (2008) The catalytic role of oxide in the thermooxidative degradation of poly(methyl methacrylate) $-\mathrm{TiO}_{2}$ nanocomposites. Polym. Degrad. Stabil., 93(6), 1131-1137.

17. Braun, R.M., Cheng, J., Parsonage, E.E., Moeller, J., Winograd, N. (2006) Depth profiling of taxol-loaded poly(styrene-b-isobutylene-b-styrene) using $\mathrm{Ga}^{+}$and $\mathrm{C}_{60}{ }^{+}$ ion beams. Appl. Surf. Sci., 252, 6615-6618. 